\title{
A High-Order Melnikov Method for Heteroclinic Orbits in Planar Vector Fields and Heteroclinic Persisting Perturbations
}

\author{
Yi Zhong (i) \\ School of Mathematical Sciences, Zhejiang University, Hangzhou, Zhejiang 310027, China \\ Correspondence should be addressed to Yi Zhong; yizhong@zju.edu.cn
}

Received 23 July 2021; Accepted 17 November 2021; Published 3 December 2021

Academic Editor: Yongqiang Fu

Copyright (c) 2021 Yi Zhong. This is an open access article distributed under the Creative Commons Attribution License, which permits unrestricted use, distribution, and reproduction in any medium, provided the original work is properly cited.

This work extends the high-order Melnikov method established by FJ Chen and QD Wang to heteroclinic orbits, and it is used to prove, under a certain class of perturbations, the heteroclinic orbit in a planar vector field that remains unbroken. Perturbations which have this property together form the heteroclinic persisting space. The Van der Pol system is analysed as an application.

\section{Introduction}

Since the pioneering work of Poincaré on celestial mechanics $[1,2]$, homoclinic tangles created by transversal intersection of the stable and unstable manifolds have been playing a prominent role in the progress of chaos theory. In 1967, Smale observed that horseshoe chaos occurs in all homoclinic tangles [3]. For the past few years, the studies in [4-7] presented that periodic sinks and $\mathrm{H} e$ nonlike attractors are also residing in homoclinic and heteroclinic tangles. The $\mathrm{H} e$ nonlike attractors provide observable chaos in the sense of Sinai-Ruelle-Bowen (SRB) measure $[8,9]$.

In general, the standard Melnikov function is enough to verify whether the homoclinic tangle or heteroclinic tangle occurs $[10,11]$. However, there exist perturbations which make the standard Melnikov function identically equal to zero. In such a case, no simple zero exists. As a result, we need to calculate high-order Melnikov functions for further investigation. There have been many previous works to refer high-order Melnikov method (see [12-19]). We focus on the method presented in [13] and extends the high-order Melnikov functions for homoclinic orbits to heteroclinic orbits.

Naturally, another question arises: does there exist a perturbation such that all the high-order Melnikov functions identically equal to zero? This means the homoclinic or heteroclinic solution remains unbroken. Through the highorder Melnikov method, we prove that the answer of the above question is positive. The union of such perturbations will be defined as heteroclinic persisting space.

This paper is built up as follows: In Section 2, we outline the main results. Section 3 is devoted to focus on the derivation of high-order Melnikov functions for heteroclinic orbits. Then, in Section 4, we prove the heteroclinic orbit remains unbroken under a certain class of autonomous perturbations. Finally, Van der Pol system is considered in Section 5 .

\section{Statement of Results}

We start with a planar system:

$$
\begin{aligned}
& \frac{\mathrm{d} x}{\mathrm{~d} t}=f(x, y), \\
& \frac{\mathrm{d} y}{\mathrm{~d} t}=g(x, y) .
\end{aligned}
$$

Assume that (1) has two hyperbolic saddle fixed points $O_{1}\left(x_{1}, y_{1}\right)$ and $O_{2}\left(x_{2}, y_{2}\right)$ with a heteroclinic solution

$$
\ell=\{(a(t), b(t)): t \in(-\infty,+\infty)\},
$$

such that 


$$
\begin{aligned}
& \lim _{t \longrightarrow-\infty}(a(t), b(t))=\left(x_{1}, y_{1}\right), \\
& \lim _{t \longrightarrow+\infty}(a(t), b(t))=\left(x_{2}, y_{2}\right) .
\end{aligned}
$$

The functions $f(x, y)$ and $g(x, y)$ are real analytic near $\ell \cup\left\{\mathrm{O}_{1}, \mathrm{O}_{2}\right\}$.

Adding time-periodic perturbations to (1) yields

$$
\begin{aligned}
& \frac{\mathrm{d} x}{\mathrm{~d} t}=f(x, y)+\varepsilon P(x, y, t), \\
& \frac{\mathrm{d} y}{\mathrm{~d} t}=g(x, y)+\varepsilon Q(x, y, t),
\end{aligned}
$$

where $\varepsilon$ is a small parameter. The periodic functions $P(x, y, t)$ and $Q(x, y, t)$ are real analytic with respect to $(x, y)$, and they are terms of second order and higher at $O_{1}$ and $O_{2}$. Let $W\left(O_{1}\right)$ be the unstable manifold of $O_{1}$ and $W_{\varepsilon}^{s}\left(\mathrm{O}_{2}\right)$ be the stable manifold of $\mathrm{O}_{2}$. In general, $W_{\varepsilon}^{u}\left(O_{1}\right) \neq W_{\varepsilon}^{s}\left(O_{2}\right)$ for $\varepsilon \neq 0$. We let $D\left(\varepsilon, t_{0}\right)$ be the splitting distance, which can be expressed in the form of

$$
D\left(\varepsilon, t_{0}\right)=M_{0}\left(t_{0}\right) \varepsilon+M_{1}\left(t_{0}\right) \varepsilon^{2}+\cdots+M_{k}\left(t_{0}\right) \varepsilon^{k+1}+\cdots,
$$

where $M_{0}\left(t_{0}\right)$ is equivalent to the standard Melnikov function in $[10,11]$ and $M_{k}\left(t_{0}\right), k=1,2, \ldots$, are the highorder Melnikov functions. Moreover, we write $a=a(t)$, $b=b(t), \dot{a}=\mathrm{d} a / \mathrm{d} t, \dot{b}=\mathrm{d} b / \mathrm{d} t, \ddot{a}=\mathrm{d} \dot{a} / \mathrm{d} t$, and $\ddot{b}=\mathrm{d} \dot{b} / \mathrm{d} t$ for easy of notations. We also introduce

$$
\begin{aligned}
& A(t, 0)=-\frac{A_{0}(t)}{\dot{a}^{2}+\dot{b}^{2}} \\
& A_{1,0}(t)=\frac{\mathrm{d} A(t, 0)}{\mathrm{d} t} \\
& A_{0,1}(t)=-\frac{A_{1}(t)}{\dot{a}^{2}+\dot{b}^{2}}+\frac{A_{0}(t) H(t)}{\left(\dot{a}^{2}+\dot{b}^{2}\right)^{2}}, \\
& B(t, 0)=\frac{B_{0}(t)}{\dot{a}^{2}+\dot{b}^{2}}, \\
& U\left(t, 0, t+t_{0}\right)=-\frac{U_{0}\left(t, t_{0}\right)}{\dot{a}^{2}+\dot{b}^{2}}, \\
& V\left(t, 0, t+t_{0}\right)=\frac{V_{0}\left(t, t_{0}\right)}{\dot{a}^{2}+\dot{b}^{2}}, \\
& U_{1,0}\left(t, t_{0}\right)=\frac{\mathrm{d} U\left(t, 0, t+t_{0}\right)}{\mathrm{d} t}, \\
& U_{0,1}\left(t, t_{0}\right)=-\frac{U_{1}\left(t, t_{0}\right)}{\dot{a}^{2}+\dot{b}^{2}}+\frac{U_{0}\left(t, t_{0}\right) H(t)}{\left(\dot{a}^{2}+\dot{b}^{2}\right)^{2}}, \\
& Z_{0}^{ \pm}\left(t, t_{0}\right)=\int_{0}^{ \pm \infty} U\left(\tau, 0, \tau+t_{0}\right) e^{\int_{0}^{\tau} A(\omega, 0) \mathrm{d} \omega} \mathrm{d} \tau \\
& S_{0}^{ \pm}\left(t, t_{0}\right)=\int_{0}^{t}\left[B(\tau, 0) Z_{0}^{ \pm}\left(\tau, t_{0}\right)+V\left(\tau, 0, \tau+t_{0}\right)\right] \mathrm{d} \tau,
\end{aligned}
$$

where 


$$
\begin{aligned}
A_{0}(t)= & {\left[f_{x}(a, b)-g_{y}(a, b)\right]\left(\dot{b}^{2}-\dot{a}^{2}\right)-2\left[f_{y}(a, b)+g_{x}(a, b)\right] \dot{a} \dot{b}, } \\
U_{0}\left(t, t_{0}\right)= & \dot{b} P\left(a, b, t+t_{0}\right)-\dot{a} Q\left(a, b, t+t_{0}\right), \\
B_{0}(t)= & {\left[f_{y}(a, b)+g_{x}(a, b)\right]\left(\dot{b}^{2}-\dot{a}^{2}\right)+2\left[f_{x}(a, b)-g_{y}(a, b)\right] \dot{a} \dot{b}, } \\
V_{0}\left(t, t_{0}\right)= & \dot{a} P\left(a, b, t+t_{0}\right)+\dot{b} Q\left(a, b, t+t_{0}\right), \\
A_{1}\left(t, t_{0}\right)= & \frac{\dot{b}}{2}\left[f_{x x}(a, b) \dot{b}^{2}+f_{y y}(a, b) \dot{a}^{2}-2 f_{x y}(a, b) \dot{a} \dot{b}\right]-\frac{a}{2}\left[g_{x x}(a, b) \dot{b}^{2}+g_{y y}(a, b) \dot{a}^{2}-2 g_{x y}(a, b) \dot{a} \dot{b}\right] \\
& -\left[f_{x}^{2}(a, b)-f_{y}^{2}(a, b)-g_{y}^{2}(a, b)+g_{x}^{2}(a, b)\right] \dot{a} \dot{b}^{2}\left[f_{x}(a, b) f_{y}(a, b)+g_{x}(a, b) g_{y}(a, b)\right]\left(\dot{a}^{2}-\dot{b}^{2}\right), \\
& -\ddot{a} P\left(a, b, t+t_{0}\right)-\ddot{b} Q\left(a, b, t+t_{0}\right), \\
U_{1}\left(t, t_{0}\right)= & \dot{a}^{2} Q_{y}\left(a, b, t+t_{0}\right)+\dot{b}^{2} P_{x}\left(a, b, t+t_{0}\right)-\left[P_{y}\left(a, b, t+t_{0}\right)+Q_{x}\left(a, b, t+t_{0}\right)\right] \dot{a} \dot{b} \\
H(t)= & \ddot{a} \ddot{b}-\ddot{a} \ddot{b} .
\end{aligned}
$$

In what follows, we give the formulas of $M_{0}\left(t_{0}\right)$ and $M_{1}\left(t_{0}\right)$.

Theorem 1. In (5), we have the following:

(i) Integral formula for $M_{0}\left(t_{0}\right)$ :

$$
M_{0}\left(t_{0}\right)=\int_{-\infty}^{+\infty} U\left(t, 0, t+t_{0}\right) e^{\int_{0}^{t} A(\omega, 0) \mathrm{d} \omega} \mathrm{d} t .
$$

(ii) Integral formula for $M_{1}\left(t_{0}\right)$ :

$$
\begin{aligned}
M_{1}\left(t_{0}\right)= & \int_{0}^{+\infty}\left[A_{1,0}(t) Z_{0}^{+}\left(t, t_{0}\right) S_{0}^{+}\left(t, t_{0}\right)+U_{1,0}\left(t, t_{0}\right) S_{0}^{+}\left(t, t_{0}\right)\right. \\
& \left.+A_{0,1}(t)\left(Z_{0}^{+}\left(t, t_{0}\right)\right)^{2}+U_{0,1}\left(t, t_{0}\right) Z_{0}^{+}\left(t, t_{0}\right)\right] e^{\int_{0}^{t} A(\omega, 0) \mathrm{d} \omega} \mathrm{d} t \\
& -\int_{0}^{-\infty}\left[A_{1,0}(t) Z_{0}^{-}\left(t, t_{0}\right) S_{0}^{-}\left(t, t_{0}\right)+U_{1,0}\left(t, t_{0}\right) S_{0}^{-}\left(t, t_{0}\right)\right. \\
& \left.+A_{0,1}(t)\left(Z_{0}^{-}\left(t, t_{0}\right)\right)^{2}+U_{0,1}\left(t, t_{0}\right) Z_{0}^{-}\left(t, t_{0}\right)\right] e^{\int_{0}^{t} A(\omega, 0) \mathrm{d} \omega} \mathrm{d} t .
\end{aligned}
$$

On the other hand, we consider the question whether there exist any perturbations such that the heteroclinic solution remains unbroken, which implies the splitting distance $D\left(\varepsilon, t_{0}\right)$ vanishes identically. By using the highorder Melnikov functions above, such class of perturbations are obtained. Before giving our main theorem, we first introduce some definitions.

Definition 1. Let $X$ be a smooth manifold and $F$ and $G$ be vector fields on it. We assume there exists a heteroclinic orbit $\ell$ of $F$ and a heteroclinic orbit $\ell_{\varepsilon}$ of $F+\varepsilon G$, where $\varepsilon$ is a small parameter. We call $G$ a heteroclinic persisting perturbation of $\ell$ if

$$
\lim _{\varepsilon \longrightarrow 0} \ell_{\varepsilon}=\ell,
$$

in the sense of Hausdorff limit.

Definition 2. All heteroclinic persisting perturbations of $\ell$ are referred to as the heteroclinic persisting space of $\ell$, which we denote by $H P(\ell)$.

It is not difficult to find a heteroclinic persisting perturbation. Let $(P(x, y, t), Q(x, y, t))$ in (4) be satisfying

$$
P(a(t), b(t), t)=Q(a(t), b(t), t)=0, \quad t \in(-\infty,+\infty) .
$$

Then, we have 


$$
\begin{aligned}
& \frac{d(a(t))}{\mathrm{d} t}=f(a(t), b(t))+\varepsilon P(a(t), b(t), t), \\
& \frac{d(b(t))}{\mathrm{d} t}=g(a(t), b(t))+\varepsilon Q(a(t), b(t), t) .
\end{aligned}
$$

It follows that $(a(t), b(t))$ is also a heteroclinic solution of (4). By Definition 1, we claim $(P(x, y, t), Q(x, y, t))$ is a heteroclinic persisting perturbation.

However, this heteroclinic persisting perturbation is trivial, since the heteroclinic orbit $(a(t), b(t))$ is just the previous one. We would like to quest for more general ones. For this purpose, we focus on an autonomous perturbed system:

$$
\begin{aligned}
& \frac{\mathrm{d} x}{\mathrm{~d} t}=f(x, y)+\varepsilon P(x, y), \\
& \frac{\mathrm{d} y}{\mathrm{~d} t}=g(x, y)+\varepsilon Q(x, y) .
\end{aligned}
$$

Then, splitting distance (5) can be rewritten as

$$
D(\varepsilon, P, Q)=M_{0}(P, Q) \varepsilon+M_{1}(P, Q) \varepsilon^{2}+\cdots+M_{k}(P, Q) \varepsilon^{k+1} \ldots,
$$

where $M_{k}(P, Q)$ is the $(k+1)$-th order Melnikov integral, $k=0,1,2, \ldots$ The following fact is trivial.

The fact is that $(P(x, y), Q(x, y))$ is a heteroclinic persisting perturbation if and only if $D(\varepsilon, P, Q)=0$ for all $|\varepsilon|<\varepsilon_{0}$, where $\varepsilon_{0}>0$ is sufficiently small.

Note that the geometric shape of heteroclinic orbits in planar vector fields are often symmetric which contributes to the computations of high-order Melnikov integrals. We obtain the following theorem.

Theorem 2. For the given vector field defined by (1), we assume $a(t)$ is odd and $b(t)$ is even in $t$ and $f(x, y)$ is even and $g(x, y)$ is odd in $x$. Then, we have
$\{(P, Q): P(x, y)$ is even in $x, Q(x, y)$ is odd in $x\} \subset H P(\ell)$.

The example considered in Section 5 will show that the parity assumption made in Theorem 2 is without loss of generality.

\section{High-Order Melnikov Method}

This section is intended to derive the high-order Melnikov functions for equation (4). The method here is based on the homoclinic case introduced in [13], but there still exist some nonnegligible differences between them.

3.1. Canonical Equation around the Heteroclinic Orbit. We introduce new phase variables $(s, z)$ by letting

$$
(x, y)=(a(s), b(s))+z(\dot{b}(s),-\dot{a}(s)) .
$$

Differentiating both sides of (16) with respect to $t$ gives

$$
\begin{aligned}
& \frac{\mathrm{d} x}{\mathrm{~d} t}=(\dot{a}(s)+z \ddot{b}(s)) \frac{\mathrm{d} s}{\mathrm{~d} t}+\dot{b}(s) \frac{\mathrm{d} z}{\mathrm{~d} t}, \\
& \frac{\mathrm{d} y}{\mathrm{~d} t}=(\dot{b}(s)-z \ddot{a}(s)) \frac{\mathrm{d} s}{\mathrm{~d} t}-\dot{a}(s) \frac{\mathrm{d} z}{\mathrm{~d} t} .
\end{aligned}
$$

By Cramer's Rule and (4), we have

$$
\begin{aligned}
& \frac{\mathrm{d} z}{\mathrm{~d} t}=-A(s, z) z-\varepsilon U(s, z, t), \\
& \frac{\mathrm{d} s}{\mathrm{~d} t}=1+B(s, z) z+\varepsilon V(s, z, t),
\end{aligned}
$$

where

$$
\begin{aligned}
A(s, z) & =-\frac{1}{z} \frac{(\dot{b}-z \ddot{a}) f(a+z \dot{b}, b-z \dot{a})-(\dot{a}+z \ddot{b}) g(a+z \dot{b}, b-z \dot{a})}{\dot{a}^{2}+\dot{b}^{2}+z(\dot{a} \vec{b}-\ddot{a} \dot{b})} \\
U(s, z, t) & =-\frac{(\dot{b}-z \ddot{a}) P(a+z \dot{b}, b-z \dot{a}, t)-(\dot{a}+z \ddot{b}) Q(a+z \dot{b}, b-z \dot{a}, t)}{\dot{a}^{2}+\dot{b}^{2}+z(\dot{a} \ddot{b}-\ddot{a} \dot{b})} \\
B(s, z) & =\frac{1}{z}\left(\frac{\dot{a} f(a+z \dot{b}, b-z \dot{a})+\dot{b} g(a+z \dot{b}, b-z \dot{a})}{\dot{a}^{2}+\dot{b}^{2}+z(\dot{a} \ddot{b}-\ddot{a} \dot{b})}-1\right) \\
V(s, z, t) & =\frac{\dot{a} P(a+z \dot{b}, b-z \dot{a}, t)+\dot{b} Q(a+z \dot{b}, b-z \dot{a}, t)}{\dot{a}^{2}+\dot{b}^{2}+z(\dot{a} \ddot{b}-\ddot{a} \dot{b})} .
\end{aligned}
$$

We suppose $(\widehat{s}(t), \widehat{z}(t))$ to be a stable solution or an unstable solution of system (18) such that $\widehat{s}\left(t_{0}\right)=0$ and $\widehat{z}\left(t_{0}\right)=z_{0}$. Let $s(t)=\widehat{s}\left(t+t_{0}\right)$ and $z(t)=\widehat{z}\left(t+t_{0}\right)$; then, $\quad(s(t), z(t)) \quad$ is well-defined for all $t \in[0,+\infty)$ or $t \in(-\infty, 0]$, and it is the solution of the system 


$$
\begin{aligned}
& \frac{\mathrm{d} z}{\mathrm{~d} t}=-A(s, z) z-\varepsilon U\left(s, z, t+t_{0}\right), \\
& \frac{\mathrm{d} s}{\mathrm{~d} t}=1+B(s, z) z+\varepsilon V\left(s, z, t+t_{0}\right),
\end{aligned}
$$

satisfying $s(0)=0$ and $z(0)=z_{0}$. To differ from the other solutions, we call them the primary stable and unstable solutions.

\subsection{Integral of Primary Solutions. We let}

$$
\begin{aligned}
& Z=\varepsilon^{-1} z, \\
& S=\varepsilon^{-1}(s-t),
\end{aligned}
$$

as one more change of variables for system (20). Then, we have

$$
\begin{aligned}
& \frac{\mathrm{d} Z}{\mathrm{~d} t}=-A(t+\varepsilon S, \varepsilon Z) Z-U\left(t+\varepsilon S, \varepsilon Z, t+t_{0}\right), \\
& \frac{\mathrm{d} S}{\mathrm{~d} t}=B(t+\varepsilon S, \varepsilon Z) Z+V\left(t+\varepsilon S, \varepsilon Z, t+t_{0}\right) .
\end{aligned}
$$

Expanding $A(t+\varepsilon S, t+\varepsilon Z), \quad U\left(t+\varepsilon S, t+\varepsilon Z, t+t_{0}\right)$, $B(t+\varepsilon S, t+\varepsilon Z)$, and $V\left(t+\varepsilon S, t+\varepsilon Z, t+t_{0}\right)$ at $(t, 0)$, we obtain

$$
\begin{aligned}
A(t+\varepsilon S, t+\varepsilon Z) & =A(t, 0)+\sum_{n+m \geq 1} A_{n, m}(t) \varepsilon^{n+m} S^{n} Z^{m}, \\
U\left(t+\varepsilon S, t+\varepsilon Z, t+t_{0}\right) & =U\left(t, 0, t+t_{0}\right)+\sum_{n+m \geq 1} U_{n, m}\left(t, t_{0}\right) \varepsilon^{n+m} S^{n} Z^{m}, \\
B(t+\varepsilon S, t+\varepsilon Z) & =B(t, 0)+\sum_{n+m \geq 1} B_{n, m}(t) \varepsilon^{n+m} S^{n} Z^{m}, \\
V\left(t+\varepsilon S, t+\varepsilon Z, t+t_{0}\right) & =V\left(t, 0, t+t_{0}\right)+\sum_{n+m \geq 1} V_{n, m}\left(t, t_{0}\right) \varepsilon^{n+m} S^{n} Z^{m} .
\end{aligned}
$$

It then follows that

$$
\begin{aligned}
& \frac{\mathrm{d} Z}{\mathrm{~d} t}=-A(t, 0) Z-U\left(t, 0, t+t_{0}\right)-\sum_{n+m \geq 1} \varepsilon^{n+m}\left[A_{n, m}(t) S^{n} Z^{m+1}+U_{n, m}\left(t, t_{0}\right) S^{n} Z^{m}\right], \\
& \frac{\mathrm{d} S}{\mathrm{~d} t}=B(t, 0) Z+V\left(t, 0, t+t_{0}\right)+\sum_{n+m \geq 1} \varepsilon^{n+m}\left[B_{n, m}(t) S^{n} Z^{m+1}+V_{n, m}\left(t, t_{0}\right) S^{n} Z^{m}\right] .
\end{aligned}
$$

We solve (24) to obtain the primary stable solution

$$
\begin{aligned}
Z^{+}\left(t, t_{0}\right)= & e^{-\int_{0}^{t} A\left(\tau_{1}, 0\right) \mathrm{d} \tau_{1}}\left(Z_{0}^{+}-\int_{0}^{t} U\left(\tau, 0, \tau+t_{0}\right) e^{\int_{0}^{\tau} A\left(\tau_{1}, 0\right) \mathrm{d} \tau_{1}} \mathrm{~d} \tau\right. \\
& -\sum_{n+m=1}^{+\infty} \varepsilon^{n+m} \int_{0}^{t}\left[A_{n, m}(\tau) Z^{+}\left(\tau, t_{0}\right)\right. \\
& \left.\left.+U_{n, m}\left(\tau, t_{0}\right)\right]\left(S^{+}\left(\tau, t_{0}\right)\right)^{n}\left(Z^{+}\left(\tau, t_{0}\right)\right)^{m} e^{\int_{0}^{\tau} A\left(\tau_{1}, 0\right) \mathrm{d} \tau_{1}} \mathrm{~d} \tau\right) \\
S^{+}\left(t, t_{0}\right)= & \int_{0}^{t}\left[V\left(\tau, 0, \tau+t_{0}\right)+B(\tau, 0) Z^{+}\left(\tau, t_{0}\right)\right] \mathrm{d} \tau \\
& +\sum_{n+m=1}^{+\infty} \varepsilon^{n+m} \int_{0}^{t}\left[B_{n, m}(\tau) Z^{+}\left(\tau, t_{0}\right)\right. \\
& \left.+V_{n, m}\left(\tau, t_{0}\right)\right]\left(S^{+}\left(\tau, t_{0}\right)\right)^{n}\left(Z^{+}\left(\tau, t_{0}\right)\right)^{m} \mathrm{~d} \tau,
\end{aligned}
$$


and the primary unstable solution

$$
\begin{aligned}
Z^{-}\left(t, t_{0}\right)= & e^{-\int_{0}^{t} A(\omega, 0) \mathrm{d} \omega}\left(Z_{0}^{-}-\int_{0}^{t} U\left(\tau, 0, \tau+t_{0}\right) e^{\int_{0}^{\tau} A\left(\tau_{1}, 0\right) \mathrm{d} \tau_{1}} \mathrm{~d} \tau\right. \\
& -\sum_{n+m=1}^{+\infty} \varepsilon^{n+m} \int_{0}^{t}\left[A_{n, m}(\tau) Z^{-}\left(\tau, t_{0}\right)\right. \\
& \left.\left.+U_{n, m}\left(\tau, t_{0}\right)\right]\left(S^{-}\left(\tau, t_{0}\right)\right)^{n}\left(Z^{-}\left(\tau, t_{0}\right)\right)^{m} e \int_{0}^{\tau} A\left(\tau_{1}, 0\right) \mathrm{d} \tau_{1} \mathrm{~d} \tau\right), \\
S^{-}\left(t, t_{0}\right)= & \int_{0}^{t}\left[V\left(\tau, 0, \tau+t_{0}\right)+B(\tau, 0) Z^{-}\left(\tau, t_{0}\right)\right] \mathrm{d} \tau \\
& +\sum_{n+m=1}^{+\infty} \varepsilon^{n+m} \int_{0}^{t}\left[B_{n, m}(\tau) Z^{-}\left(\tau, t_{0}\right)\right. \\
& \left.+V_{n, m}\left(\tau, t_{0}\right)\right]\left(S^{-}\left(\tau, t_{0}\right)\right)^{n}\left(Z^{-}\left(\tau, t_{0}\right)\right)^{m} \mathrm{~d} \tau,
\end{aligned}
$$

in which $Z_{0}^{+}$and $Z_{0}^{-}$are the initial values.

Before proceeding further, we need to consider the asymptotic behaviors of $a(t)$ and $b(t)$ as $t \longrightarrow \pm \infty$ which are crucial to get the integral equations for primary solutions. Observe that

$$
\begin{aligned}
& \lim _{t \longrightarrow-\infty}(a(t), b(t))=\left(x_{1}, y_{1}\right), \\
& \lim _{t \longrightarrow+\infty}(a(t), b(t))=\left(x_{2}, y_{2}\right),
\end{aligned}
$$

and due to the local linearization theory, there exists a small number $r>0$ such that

$$
\begin{aligned}
& \left(\begin{array}{l}
f(x, y) \\
g(x, y)
\end{array}\right)=\left(\begin{array}{ll}
f_{x}\left(x_{1}, y_{1}\right) & f_{y}\left(x_{1}, y_{1}\right) \\
g_{x}\left(x_{1}, y_{1}\right) & g_{y}\left(x_{1}, y_{1}\right)
\end{array}\right)\left(\begin{array}{c}
x-x_{1} \\
y-y_{1}
\end{array}\right)+\left(\begin{array}{l}
f_{1}(x, y) \\
g_{1}(x, y)
\end{array}\right),\left\|\left(\begin{array}{c}
x-x_{1} \\
y-y_{1}
\end{array}\right)\right\|<2 r ; \\
& \left(\begin{array}{l}
f(x, y) \\
g(x, y)
\end{array}\right)=\left(\begin{array}{ll}
f_{x}\left(x_{2}, y_{2}\right) & f_{y}\left(x_{2}, y_{2}\right) \\
g_{x}\left(x_{2}, y_{2}\right) & g_{y}\left(x_{2}, y_{2}\right)
\end{array}\right)\left(\begin{array}{l}
x-x_{2} \\
y-y_{2}
\end{array}\right)+\left(\begin{array}{l}
f_{2}(x, y) \\
g_{2}(x, y)
\end{array}\right),\left\|\left(\begin{array}{c}
x-x_{2} \\
y-y_{2}
\end{array}\right)\right\|<2 r,
\end{aligned}
$$

where $f_{1}(x, y)$ and $g_{1}(x, y)$ are terms of second order and higher at $O_{1}\left(x_{1}, y_{1}\right)$ and $f_{2}(x, y)$ and $g_{2}(x, y)$ are terms of second order and higher at $\mathrm{O}_{2}\left(x_{2}, y_{2}\right)$. We assume that $\lambda_{1}<0<\mu_{1}$ are the eigenvalues of $O_{1}\left(x_{1}, y_{1}\right)$ and $\lambda_{2}<0<\mu_{2}$ are the eigenvalues of $\mathrm{O}_{2}\left(x_{2}, y_{2}\right)$. There exist two invertible matrices $\Gamma_{1}=\left(\begin{array}{ll}\alpha_{1} & \beta_{1} \\ \gamma_{1} & \delta_{1}\end{array}\right)$ and $\Gamma_{2}=\left(\begin{array}{ll}\alpha_{2} & \beta_{2} \\ \gamma_{2} & \delta_{2}\end{array}\right)$ such that

$$
\begin{aligned}
& \Gamma_{1}\left(\begin{array}{ll}
f_{x}\left(x_{1}, y_{1}\right) & f_{y}\left(x_{1}, y_{1}\right) \\
g_{x}\left(x_{1}, y_{1}\right) & g_{y}\left(x_{1}, y_{1}\right)
\end{array}\right) \Gamma_{1}^{-1}=\left(\begin{array}{cc}
\lambda_{1} & 0 \\
0 & \mu_{1}
\end{array}\right), \\
& \Gamma_{2}\left(\begin{array}{ll}
f_{x}\left(x_{2}, y_{2}\right) & f_{y}\left(x_{2}, y_{2}\right) \\
g_{x}\left(x_{2}, y_{2}\right) & g_{y}\left(x_{2}, y_{2}\right)
\end{array}\right) \Gamma_{2}^{-1}=\left(\begin{array}{cc}
\lambda_{2} & 0 \\
0 & \mu_{2}
\end{array}\right) \text {. }
\end{aligned}
$$

In what follows, we introduce two coordinate transformations:

$$
\begin{aligned}
& \left(\begin{array}{l}
u_{1} \\
v_{1}
\end{array}\right)=\Gamma_{1}\left(\begin{array}{l}
x-x_{1} \\
y-y_{1}
\end{array}\right), \\
& \left(\begin{array}{l}
u_{2} \\
v_{2}
\end{array}\right)=\Gamma_{2}\left(\begin{array}{l}
x-x_{2} \\
y-y_{2}
\end{array}\right) .
\end{aligned}
$$

Unperturbed system (1) is transformed to

$$
\begin{aligned}
& \frac{\mathrm{d} u_{1}}{\mathrm{~d} t}=\lambda_{1} u_{1}+\tilde{f}\left(u_{1}, v_{1}\right), \\
& \frac{\mathrm{d} v_{1}}{\mathrm{~d} t}=\mu_{1} v_{1}+\tilde{g}\left(u_{1}, v_{1}\right), \\
& \frac{\mathrm{d} u_{2}}{\mathrm{~d} t}=\lambda_{2} u_{2}+\tilde{f}\left(u_{2}, v_{2}\right), \\
& \frac{\mathrm{d} v_{2}}{\mathrm{~d} t}=\mu_{2} v_{2}+\tilde{g}\left(u_{2}, v_{2}\right) .
\end{aligned}
$$

In the case $t \longrightarrow-\infty$, our problem is about the unstable solution in a small neighborhood of $O_{1}$. Let $T_{0}>0$ be 
sufficiently large. For $t<-T_{0}$, we can find a near-identity coordinate transformation:

$$
\begin{aligned}
& u_{1}=X+\sum_{j=2}^{+\infty} \phi_{j}^{1}(X, Y), \\
& v_{1}=Y+\sum_{j=2}^{+\infty} \varphi_{j}^{1}(X, Y),
\end{aligned}
$$

where $\phi_{j}^{1}(X, Y)$ and $\varphi_{j}^{1}(X, Y)$ are homogeneous polynomials of degree $j$ in $X, Y$, such that the unperturbed system near $O_{1}$ is linearized to

$$
\begin{gathered}
\frac{\mathrm{d} X}{\mathrm{~d} t}=\lambda_{1} X, \\
\frac{\mathrm{d} Y}{\mathrm{~d} t}=\mu_{1} Y .
\end{gathered}
$$

Assume that the power series in (33) are convergent on $\|(X, Y)\|<2 r$. It is not difficult to verify that

$$
\left(\begin{array}{cc}
\alpha_{1} & \beta_{1} \\
\gamma_{1} & \delta_{1}
\end{array}\right)\left(\begin{array}{c}
a(t)-x_{1} \\
b(t)-y_{1}
\end{array}\right)=\left(\begin{array}{c}
r e^{\mu_{1}\left(t-t_{0}\right)}+\sum_{j=2}^{+\infty} \phi_{j}^{1}\left(r e^{\mu_{1}\left(t-t_{0}\right)}, 0\right) \\
\sum_{j=2}^{+\infty} \varphi_{j}^{1}\left(r e^{\mu_{1}\left(t-t_{0}\right)}, 0\right)
\end{array}\right)
$$

where $t_{0}$ be such that $X\left(t_{0}\right)=r$ and $Y\left(t_{0}\right)=0$. We solve (35)

to obtain

$$
\begin{aligned}
& a(t)-x_{1}=\frac{1}{\operatorname{det}\left(\Gamma_{1}\right)}\left[-\beta_{1} r e^{\mu_{1}\left(t-t_{0}\right)}+\sum_{j=2}^{+\infty}\left(\delta_{1} \phi_{j}^{1}\left(r e^{\mu_{1}\left(t-t_{0}\right)}, 0\right)-\beta_{1} \varphi_{j}^{1}\left(r e^{\mu_{1}\left(t-t_{0}\right)}, 0\right)\right)\right], \\
& b(t)-y_{1}=\frac{1}{\operatorname{det}\left(\Gamma_{1}\right)}\left[\alpha_{1} r e^{\mu_{1}\left(t-t_{0}\right)}-\sum_{j=2}^{+\infty}\left(\gamma_{1} \phi_{j}^{1}\left(r e^{\mu_{1}\left(t-t_{0}\right)}, 0\right)-\alpha_{1} \varphi_{j}^{1}\left(r e^{\mu_{1}\left(t-t_{0}\right)}, 0\right)\right)\right] .
\end{aligned}
$$

On the other hand, let $t>T_{0}$. The problem is then about the stable solution in a small neighborhood of $\mathrm{O}_{2}$. We similarly have

$$
\begin{aligned}
& a(t)-x_{2}=\frac{1}{\operatorname{det}\left(\Gamma_{2}\right)}\left[\delta_{2} r e^{\lambda_{2}\left(t-t_{0}\right)}+\sum_{j=2}^{+\infty}\left(\delta_{2} \phi_{j}^{2}\left(r e^{\lambda_{2}\left(t-t_{0}\right)}, 0\right)-\beta_{2} \varphi_{j}^{2}\left(r e^{\lambda_{2}\left(t-t_{0}\right)}, 0\right)\right)\right] \\
& b(t)-y_{2}=\frac{1}{\operatorname{det}\left(\Gamma_{2}\right)}\left[-\gamma_{2} r e^{\lambda_{2}\left(t-t_{0}\right)}-\sum_{j=2}^{+\infty}\left(\gamma_{2} \phi_{j}^{2}\left(r e^{\lambda_{2}\left(t-t_{0}\right)}, 0\right)-\alpha_{2} \varphi_{j}^{2}\left(r e^{\lambda_{2}\left(t-t_{0}\right)}, 0\right)\right)\right]
\end{aligned}
$$

where $\phi_{j}^{2}$ and $\varphi_{j}^{2}$ are homogeneous polynomials of degree $j$.

$$
\begin{aligned}
& \lim _{t \longrightarrow-\infty} A(t, 0)>0, \\
& \lim _{t \longrightarrow+\infty} A(t, 0)<0 .
\end{aligned}
$$


Proof. We recall that

$$
A(s, z)=-\frac{1}{z} \frac{(\dot{b}-z \ddot{a}) f(a+z \dot{b}, b-z \dot{a})-(\dot{a}+z \dot{b}) g(a+z \dot{b}, b-z \dot{a})}{\dot{a}^{2}+\dot{b}^{2}+z(\dot{a} \ddot{b}-\ddot{a} \dot{b})}
$$

and let

Then,

$$
\mathscr{A}(s, z)=-z\left[\dot{a}^{2}+\dot{b}^{2}+z(\dot{a} \ddot{b}-\dot{a} \dot{b})\right] A(s, z) .
$$

$$
\begin{aligned}
\mathscr{A}(s, z)= & (\dot{b}-z \ddot{a})\left(f(a, b)+z \dot{b} f_{x}(a, b)-z \dot{a} f_{y}(a, b)+\mathcal{O}\left(z^{2}\right)\right) \\
& -(\dot{a}+z \ddot{b})\left(g(a, b)+z \dot{b} g_{x}(a, b)-z \dot{a} g_{y}(a, b)+\mathcal{O}\left(z^{2}\right)\right) \\
= & \dot{b} f(a, b)-a^{\prime} g(a, b)-z \ddot{a} f(a, b)-z \ddot{b} g(a, b)+z \dot{b}^{2} f_{x}(a, b) \\
& -z \dot{a} \dot{b} f_{y}(a, b)-z \dot{a} \dot{b} g_{x}(a, b)+z \dot{a}^{2} g_{y}(a, b)+\mathcal{O}\left(z^{2}\right) \\
= & -z \ddot{a} f(a, b)-z \ddot{b} g(a, b)+z \dot{b}^{2} f_{x}(a, b)+z \dot{a}^{2} g_{y}(a, b) \\
& -z \dot{a} \dot{b} f_{y}(a, b)-z \dot{a} \dot{b} g_{x}(a, b)+\mathcal{O}\left(z^{2}\right) .
\end{aligned}
$$

This is to imply

$$
\begin{aligned}
A(s, 0)= & \frac{((d / \mathrm{d} s) f(a, b)) f(a, b)+((d / \mathrm{d} s) g(a, b)) g(a, b)-g^{2}(a, b) f_{x}(a, b)}{f^{2}(a, b)+g^{2}(a, b)} \\
& +\frac{-f^{2}(a, b) g_{y}(a, b)+f(a, b) g(a, b)\left[f_{y}(a, b)+g_{x}(a, b)\right]}{f^{2}(a, b)+g^{2}(a, b)} \\
= & \frac{\left(f_{x} f+f_{y} g\right) f+\left(g_{x} f+g_{y} g\right) g-g^{2} f_{x}-f^{2} g_{y}+f g\left(f_{y}+g_{x}\right)}{f^{2}+g^{2}} \\
= & \frac{\left(f_{x}-g_{y}\right)\left(f^{2}-g^{2}\right)+2\left(f_{y}+g_{x}\right) f g}{f^{2}+g^{2}} .
\end{aligned}
$$

As $s<-T_{0}$, we infer from (36) that 


$$
\begin{aligned}
& f(a, b)=-\frac{1}{\operatorname{det}\left(\Gamma_{1}\right)} \beta_{1} \mu_{1} r e^{\mu_{1}\left(s-t_{0}\right)}+\mathcal{O}\left(r^{2}\right), \\
& g(a, b)=\frac{1}{\operatorname{det}\left(\Gamma_{1}\right)} \alpha_{1} \mu_{1} r e^{\mu_{1}\left(s-t_{0}\right)}+\mathcal{O}\left(r^{2}\right) .
\end{aligned}
$$

Accordingly, we have

$$
\begin{aligned}
\lim _{s \longrightarrow-\infty} A(s, 0)= & \lim _{s \longrightarrow-\infty} \frac{\left(f_{x}-g_{y}\right)\left(f^{2}-g^{2}\right)+2\left(f_{y}+g_{x}\right) f g}{f^{2}+g^{2}}(a(s), b(s)) \\
= & \lim _{s \longrightarrow-\infty}\left[\frac{\left(f_{x}(a(s), b(s))-g_{y}(a(s), b(s))\right)\left(\beta_{1}^{2}-\alpha_{1}^{2}\right) \mu_{1}^{2} r^{2} e^{2 \mu_{1}\left(s-t_{0}\right)}+\mathcal{O}\left(r^{3}\right)}{\left(\alpha_{1}^{2}+\beta_{1}^{2}\right) \mu_{1}^{2} r^{2} e^{2 \mu_{1}\left(s-t_{0}\right)}+\mathcal{O}\left(e^{3 \mu_{1}\left(s-t_{0}\right)}\right)}\right. \\
& \left.-\frac{2\left(f_{y}(a(s), b(s))+g_{x}(a(s), b(s))\right) \alpha_{1} \beta_{1} \mu_{1}^{2} r^{2} e^{2 \mu_{1}\left(s-t_{0}\right)}+\mathcal{O}\left(r^{3}\right)}{\left(\alpha_{1}^{2}+\beta_{1}^{2}\right) \mu_{1}^{2} r^{2} e^{2 \mu_{1}\left(s-t_{0}\right)}+\mathcal{O}\left(e^{3 \mu_{1}\left(s-t_{0}\right)}\right)}\right] .
\end{aligned}
$$

Recall (29), and we have

$$
\begin{aligned}
\lim _{s \longrightarrow-\infty}\left(\begin{array}{cc}
f_{x}(a(s), b(s)) & f_{y}(a(s), b(s)) \\
g_{x}(a(s), b(s)) & g_{y}(a(s), b(s))
\end{array}\right) & =\left(\begin{array}{cc}
\alpha_{1} & \beta_{1} \\
\gamma_{1} & \delta_{1}
\end{array}\right)^{-1}\left(\begin{array}{cc}
\lambda_{1} & 0 \\
0 & \mu_{1}
\end{array}\right)\left(\begin{array}{cc}
\alpha_{1} & \beta_{1} \\
\gamma_{1} & \delta_{1}
\end{array}\right) \\
& =\frac{1}{\operatorname{det}\left(\Gamma_{1}\right)}\left(\begin{array}{cc}
\lambda_{1} \alpha_{1} \delta_{1}-\mu_{1} \beta_{1} \gamma_{1} & \left(\lambda_{1}-\mu_{1}\right) \beta_{1} \delta_{1} \\
\left(\mu_{1}-\lambda_{1}\right) \alpha_{1} \gamma_{1} & -\lambda_{1} \beta_{1} \gamma_{1}+\mu_{1} \alpha_{1} \delta_{1}
\end{array}\right) .
\end{aligned}
$$

Substituting (45) into (44) gives

$$
\begin{aligned}
\lim _{s \longrightarrow-\infty} A(s, 0) & =\frac{\left(\lambda_{1}-\mu_{1}\right)\left(\alpha_{1} \delta_{1}+\beta_{1} \gamma_{1}\right)\left(\beta_{1}^{2}-\alpha_{1}^{2}\right)-2\left(\lambda_{1}-\mu_{1}\right)\left(\beta_{1} \delta_{1}-\alpha_{1} \gamma_{1}\right) \alpha_{1} \beta_{1}}{\operatorname{det}\left(\Gamma_{1}\right)\left(\alpha_{1}^{2}+\beta_{1}^{2}\right)} \\
& =\frac{\left(\lambda_{1}-\mu_{1}\right)\left(\beta_{1} \gamma_{1}-\alpha_{1} \delta_{1}\right)\left(\alpha_{1}^{2}+\beta_{1}^{2}\right)}{\operatorname{det}\left(\Gamma_{1}\right)\left(\alpha_{1}^{2}+\beta_{1}^{2}\right)} \\
& =\mu_{1}-\lambda_{1}>0 .
\end{aligned}
$$

As $s>T_{0}$, we infer from (37) that

$$
\begin{aligned}
& f(a, b)=\frac{1}{\operatorname{det}\left(\Gamma_{2}\right)} \lambda_{2} \delta_{2} r e^{\lambda_{2}\left(s-t_{0}\right)}+\mathcal{O}\left(r^{2}\right), \\
& g(a, b)=-\frac{1}{\operatorname{det}\left(\Gamma_{2}\right)} \lambda_{2} \gamma_{2} r e^{\lambda_{2}\left(s-t_{0}\right)}+\mathcal{O}\left(r^{2}\right) .
\end{aligned}
$$

After similar derivation, we have

$$
\lim _{s \longrightarrow+\infty} A(s, 0)=\lambda_{2}-\mu_{2}<0 .
$$

This completes the proof.

By using Lemma 2, one can easily verify that

$$
\lim _{t \longrightarrow-\infty} e^{-\int_{0}^{t} A\left(\tau_{1}, 0\right) \mathrm{d} \tau_{1}}=\lim _{t \longrightarrow+\infty} e^{-\int_{0}^{t} A\left(\tau_{1}, 0\right) \mathrm{d} \tau_{1}}=+\infty .
$$

Observe that $Z^{+}\left(t, t_{0}\right)$ and $Z^{-}\left(t, t_{0}\right)$ should be bounded as $t \longrightarrow \pm \infty$, and the initial values in (25) and (26) are claimed to be 


$$
\begin{aligned}
Z_{0}^{+}= & \int_{0}^{+\infty} U\left(\tau, 0, \tau+t_{0}\right) e^{\int_{0}^{\tau} A\left(\tau_{1}, 0\right) \mathrm{d} \tau_{1}} \mathrm{~d} \tau+\sum_{n+m=1}^{+\infty} \varepsilon^{n+m} \int_{0}^{+\infty}\left[A_{n, m}(\tau) Z^{+}\left(\tau, t_{0}\right)\right. \\
& \left.+U_{n, m}\left(\tau, t_{0}\right)\right]\left(S^{+}\left(\tau, t_{0}\right)\right)^{n}\left(Z^{+}\left(\tau, t_{0}\right)\right)^{m} e_{0}^{\tau} A\left(\tau_{1}, 0\right) \mathrm{d} \tau_{1} \mathrm{~d} \tau \\
Z_{0}^{-}= & \int_{0}^{-\infty} U\left(\tau, 0, \tau+t_{0}\right) e^{\int_{0}^{\tau} A\left(\tau_{1}, 0\right) \mathrm{d} \tau_{1}} \mathrm{~d} \tau+\sum_{n+m=1}^{+\infty} \varepsilon^{n+m} \int_{0}^{-\infty}\left[A_{n, m}(\tau) Z^{-}\left(\tau, t_{0}\right)\right. \\
& \left.+U_{n, m}\left(\tau, t_{0}\right)\right]\left(S^{-}\left(\tau, t_{0}\right)\right)^{n}\left(Z^{-}\left(\tau, t_{0}\right)\right)^{m} \int_{0}^{\int_{0}} A\left(\tau_{1}, 0\right) d \tau_{1} \mathrm{~d} \tau .
\end{aligned}
$$

Substituting (50) and (51) into (25) and (26), we have the integral equations of the primary stable solution

$$
\begin{aligned}
Z^{+}\left(t, t_{0}\right)= & \int_{t}^{+\infty} U\left(\tau, 0, \tau+t_{0}\right) e^{\int_{t}^{\tau} A\left(\tau_{1}, 0\right) \mathrm{d} \tau_{1}} \mathrm{~d} \tau \\
& +\sum_{n+m \geq 1} \varepsilon^{n+m} \int_{t}^{+\infty}\left[A_{n, m}(\tau) Z^{+}\left(\tau, t_{0}\right)+U_{n, m}\left(\tau, t_{0}\right)\right]\left(S^{+}\left(\tau, t_{0}\right)\right)^{n}\left(Z^{+}\left(\tau, t_{0}\right)\right)^{m} \mathrm{~d} \tau \\
S^{+}\left(t, t_{0}\right)= & \int_{0}^{t}\left[V\left(\tau, 0, \tau+t_{0}\right) \mathrm{d} \tau+\int_{0}^{t} B(\tau, 0) Z^{+}\left(\tau, t_{0}\right)\right] \mathrm{d} \tau \\
& +\sum_{n+m \geq 1} \varepsilon^{n+m} \int_{0}^{t}\left[B_{n, m}(\tau) Z^{+}\left(\tau, t_{0}\right)+V_{n, m}\left(\tau, t_{0}\right)\right]\left(S^{+}\left(\tau, t_{0}\right)\right)^{n}\left(Z^{+}\left(\tau, t_{0}\right)\right)^{m} \mathrm{~d} \tau,
\end{aligned}
$$

and the primary unstable solution

$$
\begin{aligned}
Z^{-}\left(t, t_{0}\right)= & \int_{t}^{-\infty} U\left(\tau, 0, \tau+t_{0}\right) e^{\int_{t}^{\tau} A\left(\tau_{1}, 0\right) \mathrm{d} \tau_{1}} \mathrm{~d} \tau \\
& +\sum_{n+m \geq 1} \varepsilon^{n+m} \int_{t}^{-\infty}\left[A_{n, m}(\tau) Z^{-}\left(\tau, t_{0}\right)+U_{n, m}\left(\tau, t_{0}\right)\right]\left(S^{-}\left(\tau, t_{0}\right)\right)^{n}\left(Z^{-}\left(\tau, t_{0}\right)\right)^{m} \mathrm{~d} \tau, \\
S^{-}\left(t, t_{0}\right)= & \int_{0}^{t}\left[V\left(\tau, 0, \tau+t_{0}\right) \mathrm{d} \tau+\int_{0}^{t} B(\tau, 0) Z^{-}\left(\tau, t_{0}\right)\right] \mathrm{d} \tau \\
& +\sum_{n+m \geq 1} \varepsilon^{n+m} \int_{0}^{t}\left[B_{n, m}(\tau) Z^{-}\left(\tau, t_{0}\right)+V_{n, m}\left(\tau, t_{0}\right)\right]\left(S^{-}\left(\tau, t_{0}\right)\right)^{n}\left(Z^{-}\left(\tau, t_{0}\right)\right)^{m} \mathrm{~d} \tau .
\end{aligned}
$$

3.3. Derivation of High-Order Melnikov Functions. The properties of $\left(Z^{ \pm}\left(t, t_{0}\right), S^{ \pm}\left(t, t_{0}\right)\right)$ here are coincident with the ones in [13]. We have the following power series:

$$
\begin{aligned}
& Z^{ \pm}\left(t, t_{0}\right)=Z_{0}^{ \pm}\left(t, t_{0}\right)+\sum_{k=1}^{\infty} \varepsilon^{k} Z_{k}^{ \pm}\left(t, t_{0}\right), \\
& S^{ \pm}\left(t, t_{0}\right)=S_{0}^{ \pm}\left(t, t_{0}\right)+\sum_{k=1}^{\infty} \varepsilon^{k} S_{k}^{ \pm}\left(t, t_{0}\right) .
\end{aligned}
$$


In what follows, we substitute (54) into (52) and (53) to determine $Z_{k}^{ \pm}\left(t, t_{0}\right)(k=0,1, \ldots$,$) and S_{k}^{ \pm}\left(t, t_{0}\right)(k=$ $0,1, \ldots$,$) inductively. They are$

$$
\begin{aligned}
Z_{0}^{ \pm}\left(t, t_{0}\right) & =\int_{0}^{ \pm \infty} U\left(\tau, 0, \tau+t_{0}\right) E(0, \tau) \mathrm{d} \tau \\
S_{0}^{ \pm}\left(t, t_{0}\right) & =\int_{0}^{ \pm t}\left[V(\tau, 0)+B(\tau, 0) Z_{0}^{ \pm}(\tau)\right] \mathrm{d} \tau \\
Z_{k}^{ \pm}\left(t, t_{0}\right) & =\sum_{n+m+i+\rho=k} \int_{t}^{ \pm \infty}\left[A_{n, m}(\tau) Z_{i}^{ \pm}\left(\tau, t_{0}\right)+U_{n, m}\left(\tau, t_{0}\right)\right] C_{\rho, \pm}^{n, m}\left(\tau, t_{0}\right) e^{\int_{t}^{\tau} A\left(\tau_{1}, 0\right) \mathrm{d} \tau_{1}} \mathrm{~d} \tau, \\
S_{k}^{ \pm}\left(t, t_{0}\right) & =\sum_{n+m+i+\rho=k} \int_{0}^{t}\left[B_{n, m}(\tau) Z_{i}^{ \pm}\left(\tau, t_{0}\right)+V_{n, m}\left(\tau, t_{0}\right)\right] C_{\rho, \pm}^{n, m}\left(\tau, t_{0}\right) \mathrm{d} \tau,
\end{aligned}
$$

for $k=1,2, \ldots$, . Here, $n, m, i$, and $\rho$ are nonnegative integrals, and $n+m \geq 1, \quad i \geq 0$, and $\rho \geq 0 . \quad C_{\rho,+}^{n, m}\left(t, t_{0}\right)$ and
$C_{\rho,-}^{n, m}\left(t, t_{0}\right)$ are coefficient functions of $\varepsilon^{\rho}$, which have the form of

$$
C_{\rho, \pm}^{n, m}\left(t, t_{0}\right)=\sum_{\substack{\ell_{0}+\cdots+\ell_{\rho}=n, l_{0}+\cdots+l_{\rho}=m,\left(\ell_{1}+l_{1}\right)+\cdots+\rho \cdot\left(\ell_{\rho}+l_{\rho}\right)=\rho}} K \prod_{j=0}^{\rho}\left(S_{j}^{ \pm}\left(t, t_{0}\right)\right)^{\ell_{j}} \cdot\left(Z_{j}^{ \pm}\left(t, t_{0}\right)\right)^{l_{j}} .
$$

Here, $K$ is a constant depending on the specific term. In Recall the definition of $D\left(\varepsilon, t_{0}\right)$, and we have the next section, these notations contribute to the proof of Theorem 2.

$$
\begin{aligned}
D\left(\varepsilon, t_{0}\right)=\varepsilon\left(Z^{+}\left(0, t_{0}\right)-Z^{-}\left(0, t_{0}\right)\right)=\sum_{k=0}^{+\infty} \varepsilon^{k+1}\left(Z_{k}^{+}\left(0, t_{0}\right)-Z_{k}^{-}\left(0, t_{0}\right)\right) . & \\
& \quad M_{k}\left(t_{0}\right)=Z_{k}^{+}\left(0, t_{0}\right)-Z_{k}^{-}\left(0, t_{0}\right),
\end{aligned}
$$

Then,

$$
M_{0}\left(t_{0}\right)=\int_{-\infty}^{+\infty} U\left(t, 0, t+t_{0}\right) e^{\int_{0}^{t} A(\omega, 0) \mathrm{d} \omega} \mathrm{d} t,
$$

where $Z_{k}^{+}\left(0, t_{0}\right)$ and $Z_{k}^{-}\left(0, t_{0}\right)$ are the values by letting $t=0$ in (56). Particularly, we have

and for $k=1,2, \ldots$, the $(k+1)$-th order Melnikov function is

$$
\begin{aligned}
& Z_{1}^{+}\left(0, t_{0}\right)=\sum_{n+m+i+\rho=1} \int_{0}^{+\infty}\left[A_{n, m}(\tau) Z_{i}^{+}\left(\tau, t_{0}\right)+U_{n, m}\left(\tau, t_{0}\right)\right] C_{\rho,+}^{n, m}\left(\tau, t_{0}\right) e^{\int_{0}^{\tau} A\left(\tau_{1}, 0\right) \mathrm{d} \tau_{1}} \mathrm{~d} \tau \\
& Z_{1}^{-}\left(0, t_{0}\right)=\sum_{n+m+i+\rho=1} \int_{0}^{-\infty}\left[A_{n, m}(\tau) Z_{i}^{-}\left(\tau, t_{0}\right)+U_{n, m}\left(\tau, t_{0}\right)\right] C_{\rho,-}^{n, m}\left(\tau, t_{0}\right) e^{\int_{0}^{\tau} A\left(\tau_{1}, 0\right) \mathrm{d} \tau_{1}} \mathrm{~d} \tau .
\end{aligned}
$$

Up to now, the formula of $M_{1}\left(t_{0}\right)$ can be obtained. 


\section{Heteroclinic Persisting Perturbations}

This section serves as the proof of Theorem 2. For autonomous perturbations, all the functions above having the variable $t_{0}$ are now dropped off. Before starting our proof, we need some lemmas.

Lemma 2. Under the conditions of Theorem 2, we have the following:

(1) If $n$ is odd, then $A_{n, m}(t)$ and $U_{n, m}(t)$ are even in $t$, while $B_{n, m}(t)$ and $V_{n, m}(t)$ are odd in $t$

(2) If $n$ is even, then $A_{n, m}(t)$ and $U_{n, m}(t)$ are odd in $t$, while $B_{n, m}(t)$ and $V_{n, m}(t)$ are even in $t$.

Proof. We will show the parity of $V_{n, m}(t)$, and the other three can be verified similarly. Recall that

$$
V(s, z)=\frac{\dot{a} P(a+z \dot{b}, b-z \dot{a})+\dot{b} Q(a+z \dot{b}, b-z \dot{a})}{\dot{a}^{2}+\dot{b}^{2}+z(\dot{a} \ddot{b}-\ddot{a} \dot{b})} .
$$

Since $a(s)$ is an odd function while $b(s)$ is even, then $\dot{a}(s)$ and $\ddot{b}(s)$ are even and $\dot{b}(s)$ and $\ddot{a}(s)$ are odd. It follows that

$$
V(-s, z)=V(s, z)
$$

Denote $V_{n, m}(s, z)=\partial^{n+m} V(s, z) /(\partial s)^{n}(\partial z)^{m}$. We have

$$
V_{1,0}(-s, z)=-\frac{\partial V(-s, z)}{\partial s}=-\frac{\partial V(s, z)}{\partial s}=-V_{1,0}(s, z) \text {. }
$$

Moreover, the parity in $s$ will not change by taking a derivative with respect to $z$. Inductively, we obtain

$$
V_{n, m}(s, z)= \begin{cases}-V_{n, m}(-s, z), & n \text { is odd, } \\ V_{n, m}(-s, z), & n \text { is even. }\end{cases}
$$

Since $V_{n, m}(t)$ differs from $V_{n, m}(t, 0)$ by a constant factor, the proof is now completed by letting $(s, z)=(t, 0)$.

At the end of this section, let us prove Theorem 2 with the help of the preceding preparations.

Proof of Theorem 2. To start with, we prove, for all $t \geq 0$,

$$
\begin{aligned}
& Z_{k}^{+}(t)=Z_{k}^{-}(-t), \\
& S_{k}^{+}(t)=-S_{k}^{-}(-t), \quad k \in \mathbb{N} .
\end{aligned}
$$

By using mathematical induction, we first consider the case $k=0$. Recall that

$$
\begin{aligned}
& Z_{0}^{-}(-t)=\int_{-t}^{-\infty} U(\tau, 0) e^{\int_{t}^{\tau} A\left(\tau_{1}, 0\right) \mathrm{d} \tau_{1}} \mathrm{~d} \tau, \\
& S_{0}^{-}(-t)=\int_{0}^{-t}\left[V(\tau, 0)+B(\tau, 0) Z_{0}^{-}(\tau)\right] d \tau,
\end{aligned}
$$

where $U(\tau, 0)$ is an odd function in $\tau$ and $B(\tau, 0)$ and $V(\tau, 0)$ are even functions in $\tau$. Moreover, we have

$$
e^{\int_{-t}^{-\tau} A\left(\tau_{1}, 0\right) \mathrm{d} \tau_{1}}=e^{\int_{t}^{\tau} A\left(-\tau_{1}, 0\right) \mathrm{d}\left(-\tau_{1}\right)}=e^{\int_{t}^{\tau} A\left(\tau_{1}, 0\right) \mathrm{d} \tau_{1}} .
$$

By means of variable substitution, we have

$$
\begin{aligned}
& Z_{0}^{-}(-t)=\int_{t}^{+\infty} U(-\tau, 0) e^{\int_{-t}^{-\tau} A\left(\tau_{1}, 0\right) \mathrm{d} \tau_{1}} d(-\tau)=\int_{t}^{+\infty} U(\tau, 0) e^{\int_{t}^{\tau} A\left(\tau_{1}, 0\right) \mathrm{d} \tau_{1}} \mathrm{~d} \tau=Z_{0}^{+}(t), \\
& S_{0}^{-}(-t)=\int_{0}^{t}\left[V(-\tau, 0)+B(-\tau, 0) Z_{0}^{-}(-\tau)\right] d(-\tau)=-\int_{0}^{t}\left[V(\tau, 0)+B(\tau, 0) Z_{0}^{+}(\tau)\right] \mathrm{d} \tau=-S_{0}^{+}(t) .
\end{aligned}
$$


Now, we let $L$ be a positive integer, and suppose that (66) holds for all nonnegative integer $k<L$. According to (56), we have

$$
\begin{aligned}
& Z_{L}^{-}(-t)=\sum_{n+m+i+\rho=L} \int_{-t}^{-\infty}\left[A_{n, m}(\tau) Z_{i}^{-}(\tau)+U_{n, m}(\tau)\right] C_{\rho,-}^{n, m}(\tau) e^{\int_{-t}^{\tau} A\left(\tau_{1}, 0\right) \mathrm{d} \tau_{1}} \mathrm{~d} \tau, \\
& S_{L}^{-}(-t)=\sum_{n+m+i+\rho=L} \int_{0}^{-t}\left[B_{n, m}(\tau) Z_{i}^{-}(\tau)+V_{n, m}(\tau)\right] C_{\rho,-}^{n, m}(\tau) \mathrm{d} \tau .
\end{aligned}
$$

Notice that $C_{\rho,-}^{n, m}(\tau)$ is the counterpart of $C_{\rho,+}^{n, m}(\tau)$, changing " $Z_{j}^{+}(\tau)$ " "and " $S_{j}^{+}(\tau)$ " to " $Z_{j}^{-}(\tau)$ " and " $S_{j}^{-}(\tau)$," respectively. Since $\rho<L$, we obtain

$$
\begin{aligned}
C_{\rho,-}^{n, m}(-\tau)= & \sum_{\substack{\ell_{0}+\cdots+\ell_{\rho}=n, l_{0}+\ldots+l_{\rho}=m,\left(\ell_{1}+l_{1}\right)^{+}+\cdots+\rho \cdot\left(\ell_{\rho}+l_{\rho}\right)=\rho}} K \prod_{j=0}^{\rho}\left(S_{j}^{-}(-\tau)\right)^{\ell_{j}} \cdot\left(Z_{j}^{-}(-\tau)\right)^{l_{j}} \\
= & \sum_{\substack{\ell_{0}+\cdots+\ell_{\rho}=n, l_{0}+\cdots+l_{\rho}=m,\left(\ell_{1}+l_{1}\right)^{\prime}+\cdots+\rho \cdot\left(\ell_{\rho}+l_{\rho}\right)=\rho}}^{\rho} K \prod_{j=0}^{\rho}\left(-S_{j}^{+}(\tau)^{\ell_{j}} \cdot\left(Z_{j}^{+}(\tau)^{l_{j}}\right.\right. \\
= & \sum_{\substack{\ell_{0}+\cdots+\ell_{\rho}=n, l_{0}+\cdots+l_{\rho}=m,\left(\ell_{1}+l_{1}\right)^{2}+\cdots+\rho \cdot\left(\ell_{\rho}+l_{\rho}\right)=\rho}}(-1)^{n} K \prod_{j=0}^{\rho}\left(S_{j}^{+}(\tau)\right)^{\ell_{j}} \cdot\left(Z_{j}^{+}(\tau)\right)^{l_{j}} \\
= & (-1)^{n} C_{\rho,+}^{n, m}(\tau) .
\end{aligned}
$$

That is,

$$
C_{\rho,-}^{n, m}(-\tau)= \begin{cases}-C_{\rho,+}^{n, m}(\tau), & n \text { is odd }, \\ C_{\rho,+}^{n, m}(\tau), & n \text { is even. }\end{cases}
$$

$$
\begin{aligned}
& {\left[A_{n, m}(-\tau) Z_{i}^{-}(-\tau)+U_{n, m}(-\tau)\right] C_{\rho,-}^{n, m}(-\tau) e^{\int_{-t}^{-\tau} A\left(\tau_{1}, 0\right) \mathrm{d} \tau_{1}} } \\
&=-\left[A_{n, m}(\tau) Z_{i}^{+}(\tau)+U_{n, m}(\tau)\right] C_{\rho,+}^{n, m}(\tau) e^{\int_{t}^{\tau} A\left(\tau_{1}, 0\right) \mathrm{d} \tau_{1},} \\
& {\left[B_{n, m}(-\tau) Z_{i}^{-}(-\tau)+V_{n, m}(-\tau)\right] C_{\rho,-}^{n, m}(-\tau)=\left[B_{n, m}(\tau) Z_{i}^{+}(\tau)+V_{n, m}(\tau)\right] C_{\rho,+}^{n, m}(\tau) . } \\
& \lim _{\mathcal{E} \longrightarrow 0} \ell_{\varepsilon}=\ell
\end{aligned}
$$

Finally, we obtain

$$
\begin{aligned}
Z_{L}^{-}(-t) & =Z_{L}^{+}(t), \\
S_{L}^{-}(-t) & =-S_{L}^{+}(t) .
\end{aligned}
$$

The proof of (66) is completed.

Recall (60), and for autonomous perturbation, we have $M_{k}(P, Q)=Z_{k}^{+}(0)-Z_{k}^{-}(0)=0, \quad k=0,1,2, \ldots$

Due to (14), we thus arrive the conclusion that $D(\varepsilon, P, Q)=0$ for all small $\varepsilon$. This means the stable manifold and unstable manifold coincide. As a result, there exists a heteroclinic orbit $\ell_{\varepsilon}$ which satisfies
Therefore, $(P, Q)$ is the heteroclinic persisting perturbation of $\ell$.

\section{An Concrete Example: Van der Pol System}

Let us consider the Van der Pol system on a torus:

$$
\begin{aligned}
& \frac{\mathrm{d} x}{\mathrm{~d} t}=\sin y, \\
& \frac{\mathrm{d} y}{\mathrm{~d} t}=-\sin x,
\end{aligned}
$$


where $(x, y) \in \mathbb{R}^{2}$. Without loss of generality, we fix a periodic region of the vector field in which we have four hyperbolic saddle fixed points:

$$
O_{1}(-\pi, 0), O_{2}(0, \pi), O_{3}(\pi, 0), O_{4}(0,-\pi) .
$$

We write $f(x, y)=\sin y$ and $g(x, y)=-\sin x$, and the heteroclinic solutions are

$$
\begin{aligned}
& \ell_{12}:\left\{\left(-2 \arctan \left(e^{-t}\right), \pi-2 \arctan \left(e^{-t}\right)\right), \quad t \in \mathbb{R}\right\}, \\
& \ell_{23}:\left\{\left(2 \arctan \left(e^{t}\right), \pi-2 \arctan \left(e^{t}\right)\right), \quad t \in \mathbb{R}\right\}, \\
& \ell_{34}:\left\{\left(2 \arctan \left(e^{-t}\right), 2 \arctan \left(e^{-t}\right)-\pi\right), \quad t \in \mathbb{R}\right\}, \\
& \ell_{41}:\left\{\left(-2 \arctan \left(e^{t}\right), 2 \arctan \left(e^{t}\right)-\pi\right), \quad t \in \mathbb{R}\right\} .
\end{aligned}
$$

The vector field of (77) is displayed in Figure 1.

In order to obtain the heteroclinic persisting perturbations of these heteroclinic orbits, we need some rotation transformations of coordinates to satisfy the preconditions in Theorem 2.

5.1. Persistence of $\ell_{12}$ and $\ell_{34}$. Introducing the change of variables

$$
\left(\begin{array}{c}
u_{1} \\
v_{1}
\end{array}\right)=\left(\begin{array}{cc}
\frac{\sqrt{2}}{2} & -\frac{\sqrt{2}}{2} \\
\frac{\sqrt{2}}{2} & \frac{\sqrt{2}}{2}
\end{array}\right)\left(\begin{array}{l}
x \\
y
\end{array}\right)
$$

we obtain

$$
\begin{aligned}
& \frac{\mathrm{d} u_{1}}{\mathrm{~d} t}=\frac{\sqrt{2}}{2}\left[\sin \left(\frac{u_{1}+v_{1}}{\sqrt{2}}\right)-\sin \left(\frac{u_{1}-v_{1}}{\sqrt{2}}\right)\right], \\
& \frac{\mathrm{d} v_{1}}{\mathrm{~d} t}=-\frac{\sqrt{2}}{2}\left[\sin \left(\frac{u_{1}+v_{1}}{\sqrt{2}}\right)+\sin \left(\frac{u_{1}-v_{1}}{\sqrt{2}}\right)\right] .
\end{aligned}
$$

In such a case, the saddle points are transformed to

$$
\begin{aligned}
& O_{1}^{\prime}\left(-\frac{\sqrt{2}}{2} \pi,-\frac{\sqrt{2}}{2} \pi\right), \\
& O_{2}^{\prime}\left(-\frac{\sqrt{2}}{2} \pi, \frac{\sqrt{2}}{2} \pi\right), \\
& O_{3}^{\prime}\left(\frac{\sqrt{2}}{2} \pi, \frac{\sqrt{2}}{2} \pi\right) \\
& O_{4}^{\prime}\left(\frac{\sqrt{2}}{2} \pi,-\frac{\sqrt{2}}{2} \pi\right) .
\end{aligned}
$$

Denote

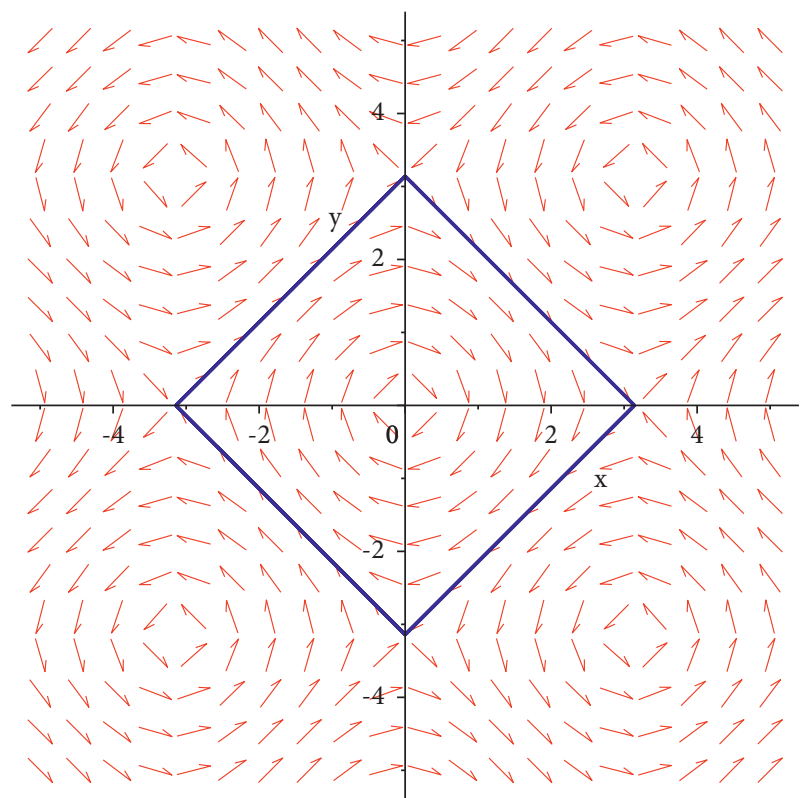

Figure 1: Vector field of (77).

$$
\begin{aligned}
& f_{1}\left(u_{1}, v_{1}\right)=\frac{\sqrt{2}}{2}\left[\sin \left(\frac{u_{1}+v_{1}}{\sqrt{2}}\right)-\sin \left(\frac{u_{1}-v_{1}}{\sqrt{2}}\right)\right], \\
& g_{1}\left(u_{1}, v_{1}\right)=-\frac{\sqrt{2}}{2}\left[\sin \left(\frac{u_{1}+v_{1}}{\sqrt{2}}\right)+\sin \left(\frac{u_{1}-v_{1}}{\sqrt{2}}\right)\right] .
\end{aligned}
$$
Figure 2.

We note that the image of $\ell_{12}$ is

$$
\ell_{12}^{\prime}:\left\{\left(u_{1}^{12}(t), v_{1}^{12}(t)\right)=\left(\frac{\sqrt{2}}{2}\left(4 \arctan \left(e^{t}\right)-\pi\right), \frac{\sqrt{2}}{2} \pi\right), \quad t \in \mathbb{R}\right\} .
$$

One can easily verify that $u_{1}^{12}(t)$ is odd in $t$ while $v_{1}^{12}(t)$ is even and $f_{1}\left(u_{1}, v_{1}\right)$ is even in $u_{1}$ while $g_{1}\left(u_{1}, v_{1}\right)$ is odd. For the convenience of derivation, we denote

$$
\Lambda\left(\ell_{12}^{\prime}\right)=\left\{\left(P_{1}, Q_{1}\right): P_{1}\left(u_{1}, v_{1}\right) \text { is even in } u_{1}, Q_{1}\left(u_{1}, v_{1}\right) \text { is odd in } u_{1}\right\} \text {. }
$$

Applying Theorem 2, we have $\Lambda\left(\ell_{12}^{\prime}\right) \subset H P\left(\ell_{12}^{\prime}\right)$. Now, we consider the following equations:

$$
\begin{aligned}
& \frac{\mathrm{d} u_{1}}{\mathrm{~d} t}=\frac{\sqrt{2}}{2}\left[\sin \left(\frac{u_{1}+v_{1}}{\sqrt{2}}\right)-\sin \left(\frac{u_{1}-v_{1}}{\sqrt{2}}\right)\right]+\varepsilon P_{1}\left(u_{1}, v_{1}\right), \\
& \frac{\mathrm{d} v_{1}}{\mathrm{~d} t}=-\frac{\sqrt{2}}{2}\left[\sin \left(\frac{u_{1}+v_{1}}{\sqrt{2}}\right)+\sin \left(\frac{u_{1}-v_{1}}{\sqrt{2}}\right)\right]+\varepsilon Q_{1}\left(u_{1}, v_{1}\right) .
\end{aligned}
$$

where $\left(P_{1}\left(u_{1}, v_{1}\right), Q_{1}\left(u_{1}, v_{1}\right)\right) \in \Lambda\left(\ell_{12}^{\prime}\right)$. Let us go back to the $(x, y)$-plane and replace $\varepsilon / \sqrt{2}$ by $\varepsilon$ for easy computation. It follows that 


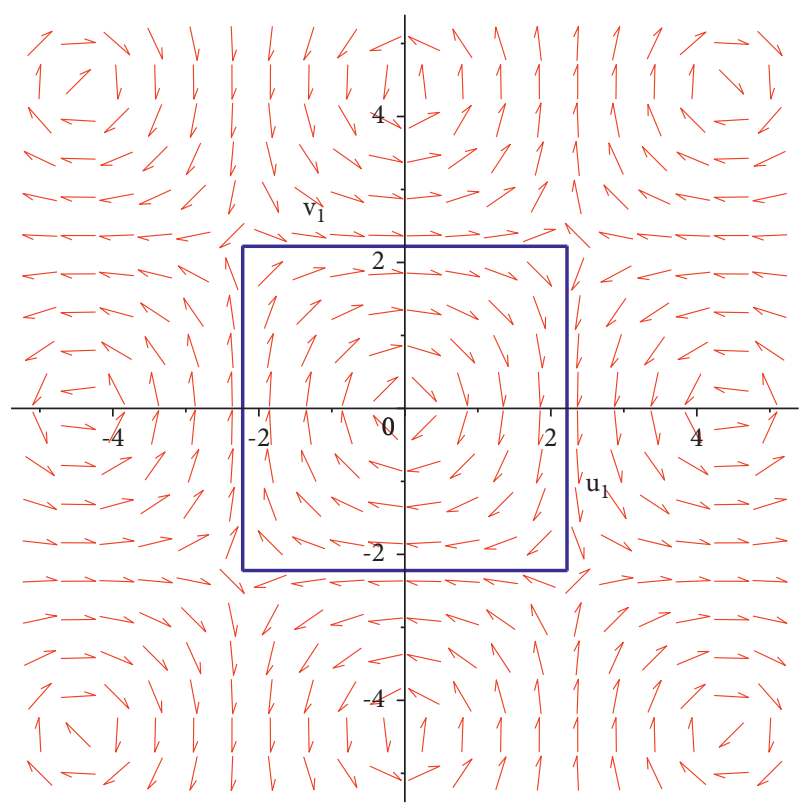

FIgURE 2: Vector field of (81).

$$
\begin{aligned}
& \frac{\mathrm{d} x}{\mathrm{~d} t}=\sin y+\varepsilon\left[P_{1}\left(\frac{x-y}{\sqrt{2}}, \frac{x+y}{\sqrt{2}}\right)+Q_{1}\left(\frac{x-y}{\sqrt{2}}, \frac{x+y}{\sqrt{2}}\right)\right] \\
& \frac{\mathrm{d} y}{\mathrm{~d} t}=-\sin x+\varepsilon\left[-P_{1}\left(\frac{x-y}{\sqrt{2}}, \frac{x+y}{\sqrt{2}}\right)+Q_{1}\left(\frac{x-y}{\sqrt{2}}, \frac{x+y}{\sqrt{2}}\right)\right] .
\end{aligned}
$$

We introduce $\Lambda\left(\ell_{12}\right)$ to be the set obtained by $\Lambda\left(\ell_{12}^{\prime}\right)$ through the transformation of coordinates (80), which means given $(P(x, y), Q(x, y)) \in \Lambda\left(\ell_{12}\right)$, there exists a $\left(P_{1}\left(u_{1}, v_{1}\right), Q_{1}\left(u_{1}, v_{1}\right)\right) \in \Lambda\left(\ell_{12}^{\prime}\right)$ such that

$$
\begin{aligned}
& P(x, y)=P_{1}\left(\frac{x-y}{\sqrt{2}}, \frac{x+y}{\sqrt{2}}\right)+Q_{1}\left(\frac{x-y}{\sqrt{2}}, \frac{x+y}{\sqrt{2}}\right), \\
& Q(x, y)=-P_{1}\left(\frac{x-y}{\sqrt{2}}, \frac{x+y}{\sqrt{2}}\right)+Q_{1}\left(\frac{x-y}{\sqrt{2}}, \frac{x+y}{\sqrt{2}}\right) .
\end{aligned}
$$

Thus, we have

$$
\Lambda\left(\ell_{12}\right)=\left\{(P, Q): \exists\left(P_{1}, Q_{1}\right) \in \Lambda\left(\ell_{12}^{\prime}\right) \text { s.t. (88) holds. }\right\} \text {. }
$$

Obviously, $\quad H P\left(\ell_{12}\right)=H P\left(\ell_{34}\right)$. We also have $\Lambda\left(\ell_{12}\right) \subset H P\left(\ell_{34}\right)$. In particular, we set

$$
\begin{aligned}
& P_{1}\left(u_{1}, v_{1}\right)=\sin \left(\frac{u_{1}+v_{1}}{\sqrt{2}}\right) \sin \left(\frac{u_{1}-v_{1}}{\sqrt{2}}\right), \\
& Q_{1}\left(u_{1}, v_{1}\right)=0 .
\end{aligned}
$$

This implies

$$
\begin{aligned}
& P(x, y)=-\sin x \sin y, \\
& Q(x, y)=\sin x \sin y .
\end{aligned}
$$

We consider the following system:

$$
\begin{aligned}
& \frac{\mathrm{d} x}{\mathrm{~d} t}=\sin y-\varepsilon \sin x \sin y, \\
& \frac{\mathrm{d} y}{\mathrm{~d} t}=-\sin x+\varepsilon \sin x \sin y .
\end{aligned}
$$

It follows that there exist two heteroclinic solutions which satisfy equality (10) in Definition 1 . The perturbed vector field and the two heteroclinic orbits $\ell_{12}^{\varepsilon}$ and $\ell_{34}^{\varepsilon}$ are plotted in Figure 3. Figure 4 shows the other two are broken.

5.2. Persistence of $\ell_{23}$ and $\ell_{41}$. For the heteroclinic orbits $\ell_{23}$ and $\ell_{41}$, we introduce another transformation of coordinates:

$$
\left(\begin{array}{l}
u_{2} \\
v_{2}
\end{array}\right)=\left(\begin{array}{cc}
\frac{\sqrt{2}}{2} & \frac{\sqrt{2}}{2} \\
-\frac{\sqrt{2}}{2} & \frac{\sqrt{2}}{2}
\end{array}\right)\left(\begin{array}{l}
x \\
y
\end{array}\right) .
$$

It follows that

$$
\begin{aligned}
& \frac{\mathrm{d} u_{2}}{\mathrm{~d} t}=\frac{\sqrt{2}}{2}\left[\sin \left(\frac{u_{2}+v_{2}}{\sqrt{2}}\right)-\sin \left(\frac{u_{2}-v_{2}}{\sqrt{2}}\right)\right], \\
& \frac{\mathrm{d} v_{2}}{\mathrm{~d} t}=-\frac{\sqrt{2}}{2}\left[\sin \left(\frac{u_{2}+v_{2}}{\sqrt{2}}\right)+\sin \left(\frac{u_{2}-v_{2}}{\sqrt{2}}\right)\right] .
\end{aligned}
$$

Denote

$$
\begin{aligned}
& f_{2}\left(u_{2}, v_{2}\right)=\frac{\sqrt{2}}{2}\left[\sin \left(\frac{u_{2}+v_{2}}{\sqrt{2}}\right)-\sin \left(\frac{u_{2}-v_{2}}{\sqrt{2}}\right)\right], \\
& g_{2}\left(u_{2}, v_{2}\right)=-\frac{\sqrt{2}}{2}\left[\sin \left(\frac{u_{2}+v_{2}}{\sqrt{2}}\right)+\sin \left(\frac{u_{2}-v_{2}}{\sqrt{2}}\right)\right] .
\end{aligned}
$$

Similarly, the image of $\ell_{23}$ is transformed to $\ell_{23}^{\prime \prime}:\left\{\left(u_{2}^{23}(t), v_{2}^{23}(t)\right)=\left(\frac{\sqrt{2}}{2}\left(\pi-4 \arctan \left(e^{-t}\right)\right), \frac{\sqrt{2}}{2} \pi\right), \quad t \in \mathbb{R}\right\}$.

The new saddle points of (94) are

$$
\begin{aligned}
& O_{1}^{\prime \prime}\left(-\frac{\sqrt{2}}{2} \pi, \frac{\sqrt{2}}{2} \pi\right), \\
& O_{2}^{\prime \prime}\left(\frac{\sqrt{2}}{2} \pi, \frac{\sqrt{2}}{2} \pi\right), \\
& O_{3}^{\prime \prime}\left(\frac{\sqrt{2}}{2} \pi,-\frac{\sqrt{2}}{2} \pi\right), \\
& O_{4}^{\prime \prime}\left(-\frac{\sqrt{2}}{2} \pi,-\frac{\sqrt{2}}{2} \pi\right) .
\end{aligned}
$$

One can easily verify that $u_{1}^{23}(t)$ is odd in $t$ while $v_{1}^{23}(t)$ is even and $f_{2}\left(u_{2}, v_{2}\right)$ is even in $u_{2}$ while $g_{2}\left(u_{2}, v_{2}\right)$ is odd. Denote 


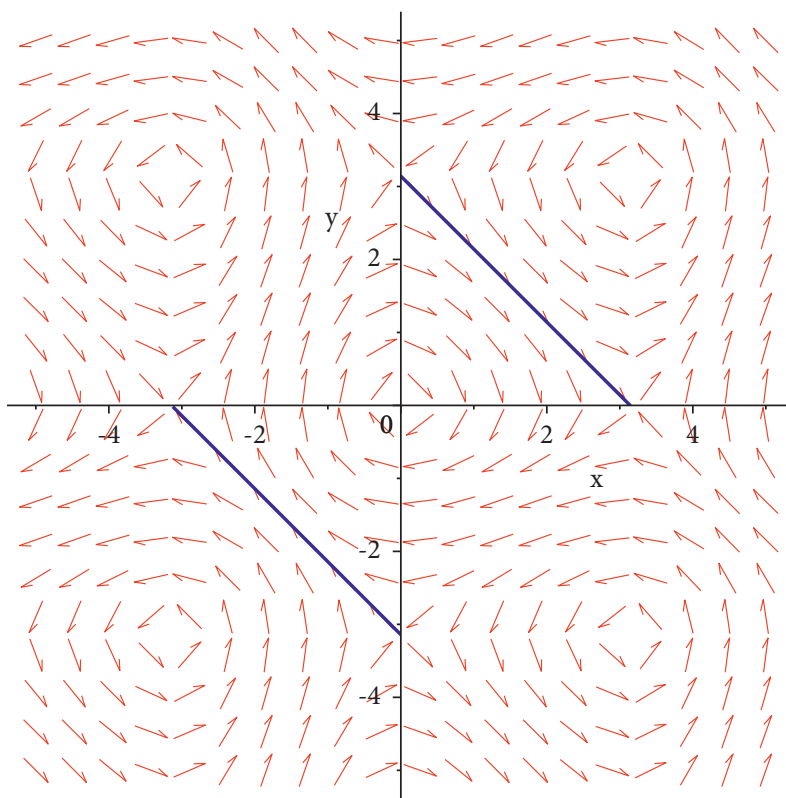

FIgURE 3: Vector field of $(92)_{\varepsilon=0.5}$ and $\ell_{12}^{\varepsilon}$ and $\ell_{34}^{\varepsilon}$.

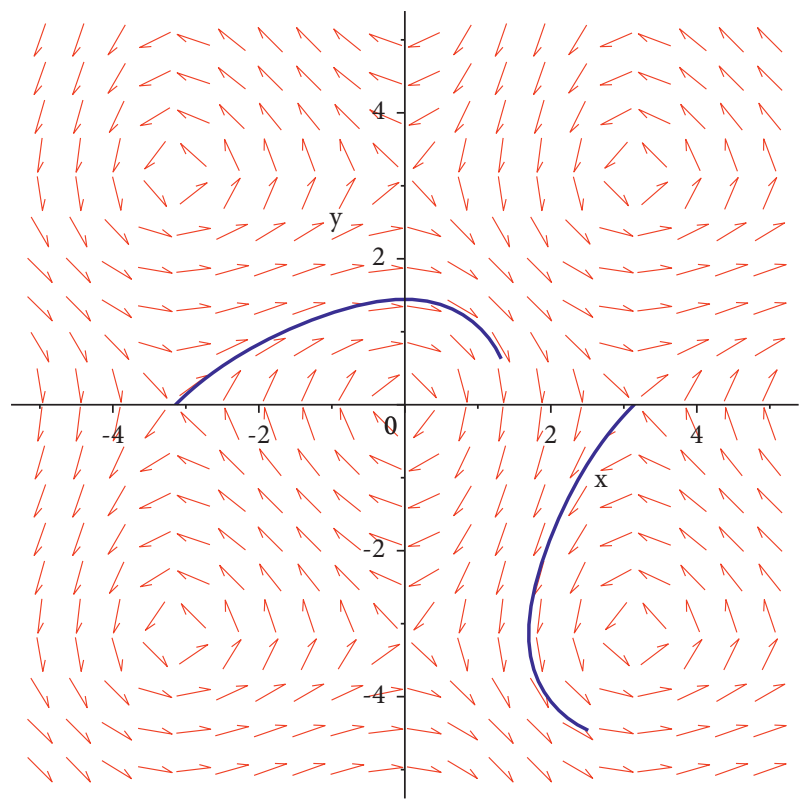

Figure 4: Vector field of $(92)_{\varepsilon=0.5}$ and $\ell_{23}^{\varepsilon}$ and $\ell_{41}^{\varepsilon}$.

$\Lambda\left(\ell_{23}^{\prime \prime}\right)=\left\{\left(P_{2}, Q_{2}\right): P_{2}\left(u_{2}, v_{2}\right)\right.$ is even in $u_{2}, Q_{2}\left(u_{2}, v_{2}\right)$ is odd in $\left.u_{2}\right\}$

It then follows from Theorem 2 that $\Lambda\left(\ell_{23}^{\prime \prime}\right) \subset H P\left(\ell_{23}^{\prime \prime}\right)$. We take $\left(P_{2}(u, v), Q_{2}(u, v)\right) \in \Lambda\left(\ell_{23}^{\prime \prime}\right)$, and consider the following perturbed system:

$$
\begin{aligned}
& \frac{\mathrm{d} u_{2}}{\mathrm{~d} t}=\frac{\sqrt{2}}{2}\left[\sin \left(\frac{u_{2}+v_{2}}{\sqrt{2}}\right)-\sin \left(\frac{u_{2}-v_{2}}{\sqrt{2}}\right)\right]+\varepsilon P_{2}\left(u_{2}, v_{2}\right), \\
& \frac{\mathrm{d} v_{2}}{\mathrm{~d} t}=-\frac{\sqrt{2}}{2}\left[\sin \left(\frac{u_{2}+v_{2}}{\sqrt{2}}\right)+\sin \left(\frac{u_{2}-v_{2}}{\sqrt{2}}\right)\right]+\varepsilon Q_{2}\left(u_{2}, v_{2}\right) .
\end{aligned}
$$

Back to $(x, y)$-plane, we have

$$
\begin{aligned}
& \frac{\mathrm{d} x}{\mathrm{~d} t}=\sin y+\varepsilon\left[P_{2}\left(\frac{x+y}{\sqrt{2}},-\frac{x-y}{\sqrt{2}}\right)-Q_{2}\left(\frac{x+y}{\sqrt{2}},-\frac{x-y}{\sqrt{2}}\right)\right], \\
& \frac{\mathrm{d} y}{\mathrm{~d} t}=-\sin x+\varepsilon\left[P_{2}\left(\frac{x+y}{\sqrt{2}},-\frac{x-y}{\sqrt{2}}\right)+Q_{2}\left(\frac{x+y}{\sqrt{2}},-\frac{x-y}{\sqrt{2}}\right)\right] .
\end{aligned}
$$

Similarly, we obtain the set $\Lambda\left(\ell_{23}\right)$ through transformation (93). Then, for any $(P(x, y), Q(x, y)) \in \Lambda\left(\ell_{23}\right)$, we obtain

$$
\begin{aligned}
& P(x, y)=P_{2}\left(\frac{x+y}{\sqrt{2}},-\frac{x-y}{\sqrt{2}}\right)-Q_{2}\left(\frac{x+y}{\sqrt{2}},-\frac{x-y}{\sqrt{2}}\right), \\
& Q(x, y)=P_{2}\left(\frac{x+y}{\sqrt{2}},-\frac{x-y}{\sqrt{2}}\right)+Q_{2}\left(\frac{x+y}{\sqrt{2}},-\frac{x-y}{\sqrt{2}}\right) .
\end{aligned}
$$

Consequently, we have

$$
\Lambda\left(\ell_{23}\right)=\left\{(P, Q): \exists\left(P_{2}, Q_{2}\right) \in C P\left(\ell_{23}^{\prime \prime}\right) \text { s.t. (101) holds }\right\} .
$$

Since $H P\left(\ell_{23}\right)=H P\left(\ell_{41}\right)$, we also have $\Lambda\left(\ell_{23}\right) \subset H P\left(\ell_{41}\right)$.

We take

$$
\begin{aligned}
& P_{2}\left(u_{2}, v_{2}\right)=\sin \left(\frac{u_{2}+v_{2}}{\sqrt{2}}\right) \sin \left(\frac{u_{2}-v_{2}}{\sqrt{2}}\right), \\
& Q_{2}\left(u_{2}, v_{2}\right)=0 .
\end{aligned}
$$

It follows that

$$
\begin{aligned}
& P(x, y)=\sin x \sin y, \\
& Q(x, y)=\sin x \sin y .
\end{aligned}
$$

Hence, we get the following perturbed system:

$$
\begin{aligned}
& \frac{\mathrm{d} x}{\mathrm{~d} t}=\sin y+\varepsilon \sin x \sin y, \\
& \frac{\mathrm{d} y}{\mathrm{~d} t}=-\sin x+\varepsilon \sin x \sin y .
\end{aligned}
$$

Figures 5 and 6 are the plots of the perturbed vector field of (105) and the two heteroclinic orbits $\ell_{23}^{\varepsilon}$ and $\ell_{41}^{\varepsilon}$. The other two are broken. 


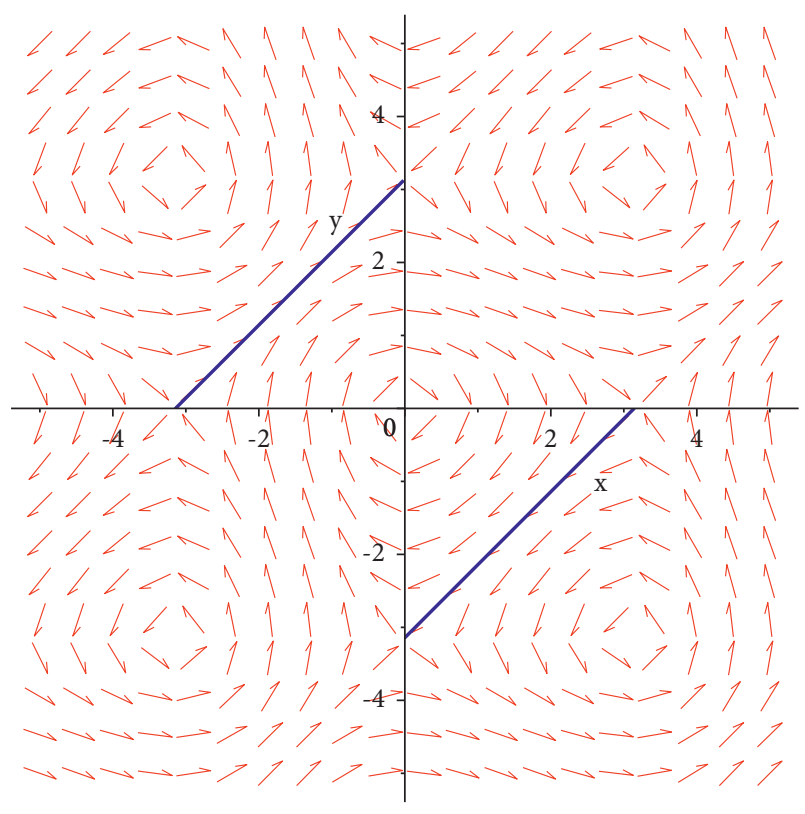

FIgURE 5: Vector field of $(105)_{\varepsilon=0.5}$ and $\ell_{23}^{\varepsilon}$ and $\ell_{41}^{\varepsilon}$.

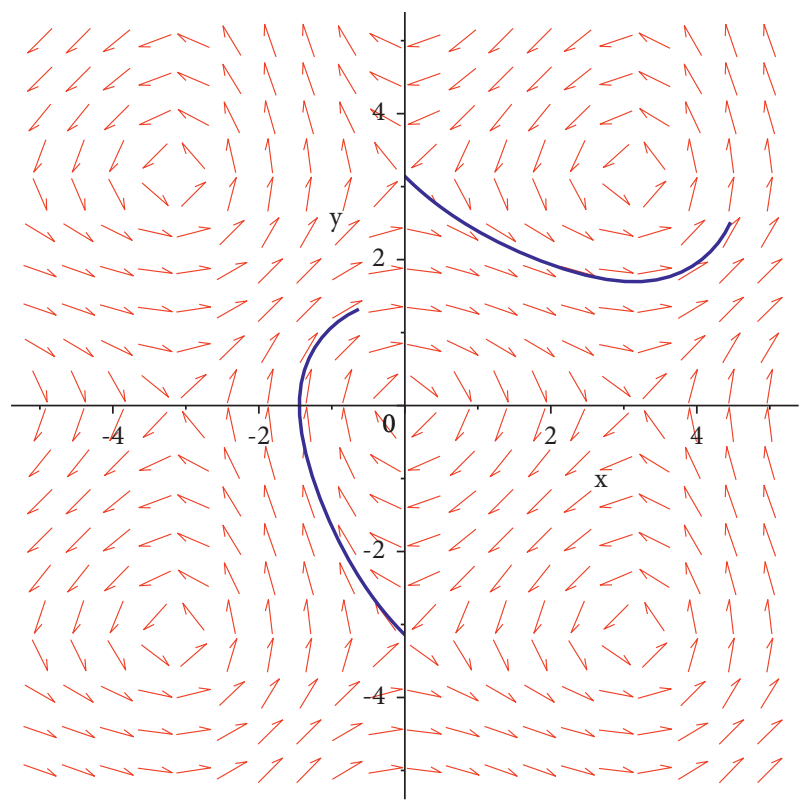

Figure 6: Vector field of $(105)_{\varepsilon=0.5}$ and $\ell_{12}^{\varepsilon}$ and $\ell_{34}^{\varepsilon}$.

5.3. Persistence of the Heteroclinic 4-Cycle. We note that in unperturbed system (77), $\quad \ell_{12} \cup \ell_{23} \cup \ell_{34} \cup \ell_{41} \cup$ $\left\{\mathrm{O}_{1}, \mathrm{O}_{2}, \mathrm{O}_{3}, \mathrm{O}_{4}\right\}$ forms a heteroclinic 4-cycle. It is natural to ask when the heteroclinic 4 -cycle remains unbroken. We rewrite $\Lambda\left(\ell_{12}\right)$ and $\Lambda\left(\ell_{23}\right)$ as

$$
\begin{gathered}
\Lambda\left(\ell_{12}\right)=\left\{(P, Q): P(x, y)=P_{1}\left(u_{1}, v_{1}\right)+Q_{1}\left(u_{1}, v_{1}\right),\right. \\
\left.Q(x, y)=-P_{1}\left(u_{1}, v_{1}\right)+Q_{1}\left(u_{1}, v_{1}\right), \quad\left(P_{1}, Q_{1}\right) \in \Lambda\left(\ell_{12}^{\prime}\right)\right\}, \\
\Lambda\left(\ell_{23}\right)=\left\{(P, Q): P(x, y)=P_{2}\left(u_{2}, v_{2}\right)-Q_{2}\left(u_{2}, v_{2}\right),\right. \\
\left.Q(x, y)=P_{2}\left(u_{2}, v_{2}\right)+Q_{2}\left(u_{2}, v_{2}\right), \quad\left(P_{2}, Q_{2}\right) \in \Lambda\left(\ell_{23}^{\prime}\right)\right\} .
\end{gathered}
$$

From (80) and (93), we have the relation between phase variables $\left(u_{1}, v_{1}\right)$ and $\left(u_{2}, v_{2}\right)$ as

$$
\left(\begin{array}{l}
u_{1} \\
v_{1}
\end{array}\right)=\left(\begin{array}{cc}
0 & -1 \\
1 & 0
\end{array}\right)\left(\begin{array}{l}
u_{2} \\
v_{2}
\end{array}\right)=\left(\begin{array}{c}
-v_{2} \\
u_{2}
\end{array}\right) \text {. }
$$

Thus, we have

$$
\begin{aligned}
& \Lambda\left(\ell_{12}\right)=\left\{(P, Q): P(x, y)=P_{1}\left(-v_{2}, u_{2}\right)+Q_{1}\left(-v_{2}, u_{2}\right),\right. \\
& \left.Q(x, y)=-P_{1}\left(-v_{2}, u_{2}\right)+Q_{1}\left(-v_{2}, u_{2}\right), \quad\left(P_{1}, Q_{1}\right) \in \Lambda\left(\ell_{12}^{\prime}\right)\right\} \\
& =\left\{(P, Q): P(x, y)=P_{1}\left(v_{2}, u_{2}\right)-Q_{1}\left(v_{2}, u_{2}\right),\right. \\
& \left.Q(x, y)=-P_{1}\left(v_{2}, u_{2}\right)-Q_{1}\left(v_{2}, u_{2}\right), \quad\left(P_{1}, Q_{1}\right) \in \Lambda\left(\ell_{12}^{\prime}\right)\right\} \text {. }
\end{aligned}
$$

Our goal is to figure out the elements in $C P\left(\ell_{12}\right) \cap C P\left(\ell_{23}\right)$ and so we let

$$
\left\{\begin{array}{l}
P_{1}\left(v_{2}, u_{2}\right)-Q_{1}\left(v_{2}, u_{2}\right)=P_{2}\left(u_{2}, v_{2}\right)-Q_{2}\left(u_{2}, v_{2}\right) \\
-P_{1}\left(v_{2}, u_{2}\right)-Q_{1}\left(v_{2}, u_{2}\right)=P_{2}\left(u_{2}, v_{2}\right)+Q_{2}\left(u_{2}, v_{2}\right) .
\end{array}\right.
$$

It then follows that

$$
\left\{\begin{array}{l}
P_{1}\left(v_{2}, u_{2}\right)=-Q_{2}\left(u_{2}, v_{2}\right) \\
Q_{1}\left(v_{2}, u_{2}\right)=-P_{2}\left(u_{2}, v_{2}\right) .
\end{array}\right.
$$

Depending on the parities of $P_{1}, P_{2}, Q_{1}$, and $Q_{2}$ with respect to the first variable, we can use (110) to obtain their parities with respect to the second variable. Let

$$
\begin{gathered}
\Omega=\left\{\left(P_{2}, Q_{2}\right): P_{2}\left(u_{2}, v_{2}\right) \text { is even in } u_{2} \text { and odd in } v_{2},\right. \\
\left.Q_{2}\left(u_{2}, v_{2}\right) \text { is odd in } u_{2} \text { and even in } v_{2}\right\} .
\end{gathered}
$$

We finally get

$$
\begin{array}{r}
\Lambda\left(\ell_{12}\right) \cap \Lambda\left(\ell_{23}\right)=\left\{(P, Q): P(x, y)=P_{2}\left(u_{2}, v_{2}\right)-Q_{2}\left(u_{2}, v_{2}\right),\right. \\
\left.Q(x, y)=P_{2}\left(u_{2}, v_{2}\right)+Q_{2}\left(u_{2}, v_{2}\right), \quad\left(P_{2}, Q_{2}\right) \in \Omega\right\},
\end{array}
$$

in which $u_{2}=\sqrt{2} / 2(x+y)$ and $v_{2}=-\sqrt{2} / 2(x-y)$.

In order to visualize this result, we consider the following perturbations: 


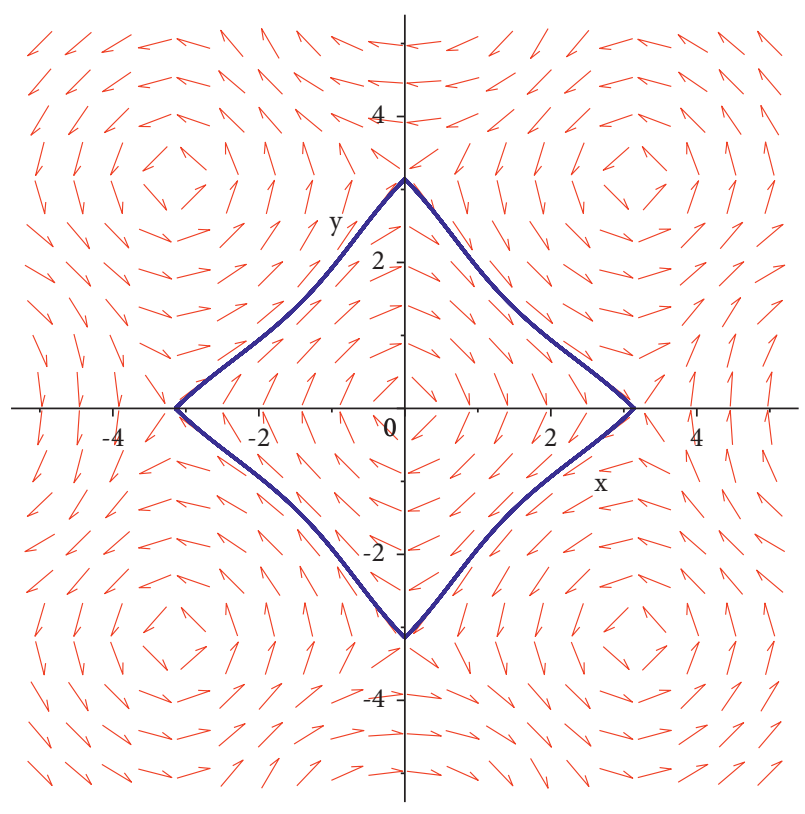

Figure 7: Vector field of $(105)_{\varepsilon=0.5}$.

$$
\begin{gathered}
P(x, y)=\sin x \sin y\left[\sin \left(\frac{x-y}{2}\right)+\sin \left(\frac{x+y}{2}\right)\right] \\
Q(x, y)=\sin x \sin y\left[\sin \left(\frac{x-y}{2}\right)-\sin \left(\frac{x+y}{2}\right)\right] .
\end{gathered}
$$

Adding them to system (77) gives

$$
\begin{aligned}
& \frac{\mathrm{d} x}{\mathrm{~d} t}=\sin y+\varepsilon \sin x \sin y\left[\sin \left(\frac{x-y}{2}\right)+\sin \left(\frac{x+y}{2}\right)\right], \\
& \frac{\mathrm{d} y}{\mathrm{~d} t}=-\sin x+\varepsilon \sin x \sin y\left[\sin \left(\frac{x-y}{2}\right)-\sin \left(\frac{x+y}{2}\right)\right] .
\end{aligned}
$$

Since $(P(x, y), Q(x, y)) \in \Lambda\left(\ell_{12}\right) \cap \Lambda\left(\ell_{23}\right)$, all the four heteroclinic orbits of (114) remain unbroken (see Figure 7).

\section{Data Availability}

All data included in this study are available upon request to the author.

\section{Conflicts of Interest}

The author declares no conflicts of interest.

\section{References}

[1] H. Poincaré, "Sur le probl $e$ me des trois corpset les e quations de la dynamique," Acta Mathematica, vol. 13, pp. 1-270, 1890.

[2] H. Poincaré, Les Méthodes Nouvelles de la Mécanique Céleste, Gauthiers-Villars, Paris, France, 1892.

[3] S. Smale, "Differentiable dynamical systems," Bulletin of the American Mathematical Society, vol. 73, no. 6, pp. 747-817, 1967.

[4] F. Chen, A. Oksasoglu, and Q. Wang, "Heteroclinic tangles in time-periodic equations," Journal of Differential Equations, vol. 254, no. 3, pp. 1137-1171, 2013.
[5] Q. D. Wang and A. Oksasoglu, "Rank one chaos: theory and applications," International Journal of Bifurcation and Chaos, vol. 18, no. 5, pp. 1261-1319, 2008.

[6] Q. D. Wang and A. Oksasoglu, "Periodic occurrence of chaotic behavior of homoclinic tangles," Physica D: Nonlinear Phenomena, vol. 239, no. 7, pp. 387-395, 2010.

[7] Q. Wang and A. Oksasoglu, "Dynamics of homoclinic tangles in periodically perturbed second-order equations," Journal of Differential Equations, vol. 250, no. 2, pp. 710-751, 2011.

[8] Q. Wang and L.-S. Young, "Toward a theory of rank one attractors," Annals of Mathematics, vol. 167, no. 2, pp. 349-480, 2008.

[9] L.-S. Young, "What are SRB measures, and which dynamical systems have them?" Journal of Statistical Physics, vol. 108, pp. 733-754, 2002.

[10] J. Guckenheimer and P. Holmes, Nonlinear Oscillations, Dynamical Systems and Bifurcations of Vector Fields, Springer, New York, NY, USA, 1983.

[11] V. K. Melnikov, "On the stability of the center for time periodic perturbations," Transactions of the Moscow Mathematical Society, vol. 12, pp. 1-57, 1963.

[12] A. Buica, A. Gasull, and J. Yang, "The third order Melnikov function of a quadratic center under quadratic perturbations," Journal of Mathematical Analysis and Applications, vol. 331, no. 1, pp. 443-454, 2007.

[13] F. Chen and Q. Wang, "High-order Melnikov method for time-periodic equations," Advanced Nonlinear Studies, vol. 17, no. 4, pp. 793-818, 2017.

[14] F. Chen and Q. Wang, "High order Melnikov method: theory and application," Journal of Differential Equations, vol. 267, no. 2, pp. 1095-1128, 2019.

[15] S.-T. Cristina and J. K. Tasso, "Higher-order Melnikov theory for adiabatic systems," Journal of Mathematics and Physics, vol. 37, no. 12, pp. 6220-6249, 1996.

[16] I. D. Iliev, "Higher order Melnikov functions for degenerate cubic Hamiltonians," Advances in Differential Equations, vol. 1, no. 4, pp. 689-708, 1996.

[17] I. D. Iliev and L. M. Perko, "Higher order bifurcations of limit cycles," Journal of Differential Equations, vol. 154, no. 2, pp. 339-363, 1999.

[18] K. Yagasaki, "Higher-order Melnikov method and chaos for two-degree-of-freedom Hamiltonian systems with saddlecenters," Discrete \& Continuous Dynamical Systems - A, vol. 29, no. 1, pp. 387-402, 2011.

[19] Zh. F. Zhang and B. Y. Li, "High order Melnikov functions and the problem of uniformity in global bifurcation," Annali di Matematica Pura ed Applicata, vol. 161, no. 4, pp. 181-212, 1992. 